\title{
A scale-dependent Lagrangian dynamic model for large eddy simulation of complex turbulent flows
}

\author{
Elie Bou-Zeid ${ }^{\text {a) }}$ \\ Department of Geography and Environmental Engineering and Center for Environmental \\ and Applied Fluid Mechanics, Johns Hopkins University, 313 Ames Hall, 3400 North Charles Street, \\ Baltimore, Maryland 21218 \\ Charles Meneveau ${ }^{\text {b) }}$ \\ Department of Mechanical Engineering and Center for Environmental and Applied Fluid Mechanics, \\ Johns Hopkins University, 127 Latrobe Hall, 3400 North Charles Street, Baltimore, Maryland 21218 \\ Marc Parlange $e^{\mathrm{c})}$ \\ School of Architecture, Civil, and Environmental Engineering, Swiss Federal Institute of Technology \\ at Lausanne, Building GC-Ecublens, CH-1015 Lausanne, Switzerland
}

(Received 25 August 2004; accepted 26 October 2004; published online 19 January 2005)

\begin{abstract}
A scale-dependent dynamic subgrid model based on Lagrangian time averaging is proposed and tested in large eddy simulations (LES) of high-Reynolds number boundary layer flows over homogeneous and heterogeneous rough surfaces. The model is based on the Lagrangian dynamic Smagorinsky model in which required averages are accumulated in time, following fluid trajectories of the resolved velocity field. The model allows for scale dependence of the coefficient by including a second test-filtering operation to determine how the coefficient changes as a function of scale. The model also uses the empirical observation that when scale dependence occurs (such as when the filter scale approaches the limits of the inertial range), the classic dynamic model yields the coefficient value appropriate for the test-filter scale. Validation tests in LES of high Reynolds number, rough wall, boundary layer flow are performed at various resolutions. Results are compared with other eddy-viscosity subgrid-scale models. Unlike the Smagorinsky-Lilly model with wall-damping (which is overdissipative) or the scale-invariant dynamic model (which is underdissipative), the scale-dependent Lagrangian dynamic model is shown to have good dissipation characteristics. The model is also tested against detailed atmospheric boundary layer data that include measurements of the response of the flow to abrupt transitions in wall roughness. For such flows over variable surfaces, the plane-averaged version of the dynamic model is not appropriate and the Lagrangian averaging is desirable. The simulated wall stress overshoot and relaxation after a jump in surface roughness and the velocity profiles at several downstream distances from the jump are compared to the experimental data. Results show that the dynamic Smagorinsky coefficient close to the wall is very sensitive to the underlying local surface roughness, thus justifying the use of the Lagrangian formulation. In addition, the Lagrangian formulation reproduces experimental data more accurately than the planar-averaged formulation in simulations over heterogeneous rough walls. (C) 2005 American Institute of Physics. [DOI: 10.1063/1.1839152]
\end{abstract}

\section{INTRODUCTION}

Large-eddy simulation (LES) has become an important tool for the study of high-Reynolds number environmental ${ }^{1-8}$ and engineering ${ }^{9-11}$ turbulent flows. LES resolves the flow at scales larger than a certain size $\Delta$, while the smaller scales are parametrized. The classic, most often used parametrization (the Smagorinsky model ${ }^{12}$ ) is based on the concepts of eddy-viscosity and mixing length, in which the subgrid-scale

\footnotetext{
${ }^{\text {a) } T e l e p h o n e: ~ 1-410-516-5031 . ~ F a x: ~ 1-410-516-8996 . ~ E l e c t r o n i c ~ m a i l: ~}$ eliebz@jhu.edu

b) Telephone: 1-410-516-7082. Fax: 1-410-516-7254. Electronic mail: meneveau@jhu.edu

${ }^{c)}$ Also at Department of Geography and Environmental Engineering and Center for Environmental and Applied Fluid Mechanics, Johns Hopkins University, Baltimore, MD 21218. Telephone: +41-21-693-6391. Electronic mail: Marc.Parlange@epfl.ch
}

(SGS) stress tensor (its traceless part) is modeled according to

$$
\tau_{i j}^{\mathrm{SMAG}}=-2 \nu_{T} \widetilde{S}_{i j}=-2\left(c_{s, \Delta} \Delta\right)^{2}|\widetilde{S}| \widetilde{S}_{i j} .
$$

Above, $\nu_{T}$ is the eddy viscosity, $\widetilde{S}_{i j}=0.5\left(\partial_{j} \widetilde{u}_{i}+\partial_{i} \widetilde{u}_{j}\right)$ is the resolved strain rate tensor (where $\widetilde{u}_{i}$ is the resolved velocity field), and the strain-rate magnitude is given by $|\widetilde{S}|$ $=\sqrt{2 \widetilde{S}_{i j} \widetilde{S}_{i j}}$. The only undetermined parameter in the above expression is the Smagorinsky coefficient $c_{s, \Delta}$. Even though many SGS models that do not rely on the viscous analogy concept have been developed, this paper will focus on eddyviscosity models since they continue to be the most widely used in practice, either individually or in conjunction with the similarity type models in the so-called mixed models. ${ }^{13-18}$ 
In 1991, an important development took place in LES with the introduction of the dynamic model and the Germano identity. ${ }^{19,20}$ By relating stresses at different scales, the Germano identity allows unknown model coefficients, such as $c_{s, \Delta}$, to be determined from the smallest resolved scales between the grid-scale $\Delta$ and a test-filter scale $\alpha \Delta(\alpha>1$, usually $\alpha=2$ ). A major assumption of the original dynamic approach is scale-similarity, i.e., that model coefficients are the same at different scales: $c_{s, \Delta}=c_{s, \alpha \Delta}$ (see the discussion by Meneveau and $\mathrm{Katz}^{21}$ ). Scale invariance is a reasonable assumption if $\Delta$ pertains to an idealized inertial range of turbulence, but it is not expected to hold if $\Delta$ falls near a transition scale that separates different physical processes occurring in distinct ranges of scales. One example where $\Delta$ falls near a transition scale occurs when the grid scale $\Delta$ approaches the integral scale, a limit that is of relevance when LES approaches the Reynolds-averaged Navier-Stokes formulation in certain parts of the flow. Of specific interest to applications to be examined in this paper, such a situation occurs in LES of high-Reynolds number wall-bounded flows where the integral scale is on the order of the distance to the wall. At the high-Reynolds numbers that occur in applications to atmospheric or oceanic boundary layers, LES cannot resolve the viscous sublayer (due to computational power limitations). In such applications, the first few cells near the surface have a grid scale on the order of the local integral scale and inaccurate results are obtained from the traditional scale-invariant dynamic model (see SGS comparison section in this paper and Refs. 22 and 23 for a discussion and illustration of this effect). Moreover, in this situation the subgrid stresses carry a significant fraction of the total mean momentum fluxes, and hence the LES results are particularly sensitive to the SGS model.

To address this shortcoming of the traditional dynamic model, Porté-Agel et al. ${ }^{22}$ proposed a scale-dependent version of the dynamic model in which a second test filter determines how the coefficient changes across scales, thus providing more accurate estimation of the coefficient at the grid scale. In the tests of atmospheric boundary layer flow over homogeneous surfaces performed in Porté-Agel et al. ${ }^{22}$ the scale-dependent dynamic model was implemented using planar averaging, i.e., the averages required to enforce the Germano identity were evaluated over horizontal planes parallel to the ground. This was appropriate for the simple geometries envisioned in those tests, where horizontal planes correspond to directions of statistical homogeneity of the turbulence. An important question is how to treat complexgeometry flows that do not possess directions of statistical homogeneity and thus do not present obvious spatial domains over which to evaluate averages during LES.

For the dynamic model, the issue of averaging the terms in the Germano identity has been the subject of considerable research $^{19,24,25}$ (also see discussion in Pope ${ }^{26}$ ). Especially in the context of eddy-viscosity closures, averaging is crucial to reduce the large amount of noise that is present when no averaging is performed (recall that in LES one must evaluate the divergence of the modeled SGS stress tensor; therefore, unphysical fluctuations in the coefficient can lead to significant errors, not to mention numerical instability if the coef- ficient becomes negative). To reduce the noise for applications in complex geometry flow, time averaging was proposed by Meneveau et al. ${ }^{24}$ In order to comply with Galilean invariance, time must be considered by following fluid parcels of the flow. Thus the Lagrangian dynamic model was proposed and tested in a number of flows. ${ }^{15,16,24,27-30}$ In these applications, viscous sublayers were resolved and hence the assumption of scale invariance was justified; the scaleinvariant Lagrangian approach allowed determination of the coefficient in these complex-geometry situations. However, for applications where the viscous sublayer cannot be resolved, such as high-Reynolds number (e.g., atmospheric) boundary layers over complex terrain, that model is not applicable due to the lack of scale invariance near the ground.

Thus, an important issue remains, namely, the formulation of a scale-dependent Lagrangian dynamic model. This paper is devoted to this task. In Sec. II we review the basic elements of the scale-invariant dynamic model, the scaledependent dynamic formulation with planar averaging of Porté-Agel et al., ${ }^{22}$ and the Lagrangian scale-invariant dynamic model of Meneveau et al. ${ }^{24}$ Section III of the paper presents the proposed Lagrangian scale-dependent dynamic model, enabling applications of the dynamic model to complex-geometry flows without assuming scale-invariance or spatial homogeneity. Section IV describes the numerical code used in this work to simulate high-Reynolds number boundary-layer flows and presents test results in horizontally homogeneous flows for which the performances of the various SGS models described in Secs. II and III can be compared in detail. Section V describes applications of the Lagrangian scale-dependent dynamic model to high-Reynolds number atmospheric flow over rough surfaces with abrupt changes in wall roughness (i.e., horizontally nonhomogeneous), and compares the results to existing field measurements data and to the results obtained with a planar-averaged version of the scale-dependent model. Conclusions are presented in Sec. VI.

\section{REVIEW OF THE DYNAMIC, SCALE-DEPENDENT, AND LAGRANGIAN DYNAMIC SGS MODELS}

The original nondynamic Smagorinsky-Lilly model (denoted SMAG below) has already been introduced in Sec. I. For isotropic homogeneous turbulence, with $\Delta$ falling in the inertial range, the analysis of Lilly ${ }^{31}$ yields $c_{s} \sim 0.16$ (for the spectral cutoff filter), a value that provides good results in LES of idealized isotropic turbulence. It remains to point out that for applications to high-Reynolds number boundary layers in which the viscous sublayer is not resolved (see Pope ${ }^{26}$ ) a wall-damping function needs to be included in the specification of the coefficient, otherwise turbulence generation is excessively damped and insufficient kinetic energy occurs in the resolved scales of the simulation. A classic wall-damping function was proposed by Mason and Thompson ${ }^{32}$ where the SGS mixing length, $\lambda=c_{s, \Delta} \Delta$ is decreased close to the surface to merge smoothly with the $\lambda \sim z$ behavior expected there. The resulting damping function is 


$$
\frac{1}{\lambda^{n}}=\frac{1}{\lambda_{0}^{n}}+\frac{1}{\left[\kappa\left(z+z_{o}\right)\right]^{n}},
$$

where $\kappa$ is the von-Karman constant $(\approx 0.4)$ and $\lambda_{0}=c_{s, 0} \Delta$ is the mixing length away from the wall (in a region of nearly homogeneous isotropic turbulence). In simulations using the Lilly-Smagorinsky model in this study, $c_{s, 0}$ is taken as 0.16 and a value of 2 is assigned to $n$, which fixes the damping function shape (alternative values of $n=1$ and $c_{s, 0}=0.1$ were also tested in other studies, see Porté-Agel et al. ${ }^{22}$ for more details).

Despite the use of this wall-damping function (with varying values of $c_{s, 0}$ and $n$ ), the Smagorinsky-Lilly model remains overdissipative $\mathrm{e}^{22,32}$ and would require further detailed calibrations of the coefficient to yield more accurate results. To avoid the need for such case-by-case calibration of parameters, the dynamic model of Germano et al. ${ }^{19}$ was proposed.

\section{A. The dynamic model}

The dynamic model ${ }^{19}$ consists of using the smallest resolved scales to measure the model coefficient during the simulation. The model is based on a relation between SGS stresses at different scales (the grid scale $\Delta$ and a test filter scale $\alpha \Delta$, where $\alpha$ is usually taken as 2) expressed by the following identity:

$$
L_{i j}=T_{i j}-\bar{\sigma}_{i j}=\overline{\tilde{u}_{i} \tilde{u}_{j}}-\overline{\tilde{u}}_{i} \overline{\tilde{u}}_{j} .
$$

Here $\sigma_{i j}$ is the SGS stress tensor at scale $\Delta, T^{i j}$ is the SGS stress tensor at the test-filter scale $\alpha \Delta$ and $L^{i j}$ is the SGS stress tensor defined from scales intermediate between $\Delta$ and $\alpha \Delta . L^{i j}$ is the resolved stress tensor and can be computed exactly from the resolved velocity field using Eq. (3). Throughout the paper, a tilde $(\sim)$ denotes the filtering operation at the grid-scale $\Delta$ and a bar $\left(^{-}\right)$denotes test-filtering at the test-filter scale $\alpha \Delta$, typically $\alpha=2$. Later on, a caret $\left(^{(}\right)$ denotes the second test-filtering at a second test-filter scale $\alpha^{2} \Delta$. Ensemble averaging will be denoted by brackets \langle\rangle . Brackets followed by dimensions subscripts will denote averaging in all the indicated dimensions; for example, $\langle u\rangle_{x, y}$ is the velocity averaged over the $x$ and $y$ directions.

Using the Smagorinsky model to express the deviatoric parts of SGS stresses at the scales $\Delta$ and $\alpha \Delta$ and assuming that the coefficient $c_{s, \Delta}$ does not fluctuate strongly in space to justify extracting it from the test-filtering operation ${ }^{24,25}$ results in the following expressions:

$$
\bar{\tau}_{i j}=\bar{\sigma}_{i j}^{D}=-2 c_{s, \Delta}^{2} \Delta^{2}\left|\overline{\widetilde{S} \mid \widetilde{S}_{i j}}, \quad T_{i j}^{D}=-2 c_{s, \alpha \Delta}^{2}(\alpha \Delta)^{2}\right| \overline{\tilde{S}} \mid \overline{\tilde{S}}_{i j}
$$

The superscript $D$ denotes the deviatoric (trace-free) part of the tensor. Replacing in Eq. (3) yields an error in that identity induced by the use of the Smagorinsky model. This error is

$$
e_{i j}=L_{i j}^{D}-\left(T_{i j}^{D}-\bar{\tau}_{i j}\right)=L_{i j}^{D}-c_{s, \Delta}^{2} M_{i j},
$$

where $M_{i j}$ is given by

$$
M_{i j}=2 \Delta^{2}\left[\overline{|\widetilde{S}| \widetilde{S}_{i j}}-\alpha^{2} \beta|\overline{\tilde{S}}| \tilde{\tilde{S}}_{i j}\right] ;
$$

$\beta=c_{s, \alpha \Delta}^{2} / c_{s, \Delta}^{2}$ is a parameter that accounts for possible scale dependence of $c_{s, \Delta}$.
Usually, the use of this model makes the assumption of scale invariance, i.e., $c_{s, \Delta}=c_{s, \alpha \Delta}$ or $\beta=1$. To obtain an optimal value of $c_{s, \Delta}$, the square error $e_{i j} e_{i j}$, is minimized contracted over all tensor terms. ${ }^{33}$ Nevertheless, the local determination of $c_{s, \Delta}$ at every grid point yields a highly variable coefficient that is numerically unstable, mainly due to a high frequency of negative values. Some averaging is hence needed to stabilize the coefficient. Ghosal ${ }^{25}$ showed that averaging over homogeneous spatial directions yields a system that is consistent with the extraction of $c_{s, \Delta}$ from the filter operation and that is equivalent to Lilly's expression averaged over homogeneous directions. Interestingly, Pope also showed that if the coefficient is optimized to minimize (in a least-square sense) the dependence of relevant turbulence statistics on the grid scale $\Delta$, the expression obtained is again exactly Lilly's expression. ${ }^{34}$

In wall-bounded flows, horizontal planes are usually selected as the homogeneous directions for averaging. In the absence of homogeneous directions, the Lagrangian approach $^{24}$ can be used to average the coefficient over time along fluid pathlines (the approach is further explained in Sec. II C below). However, for any type of averaging, denoted by brackets \langle\rangle , the Smagorinsky coefficient determined by a least-square error minimization of $\left\langle e_{i j} e_{i j}\right\rangle$ can be written as

$$
c_{s, \Delta}^{2}=\frac{\left\langle L_{i j} M_{i j}\right\rangle}{\left\langle M_{i j} M_{i j}\right\rangle} .
$$

Note that the contraction of $L_{i j}$ with $M_{i j}$ eliminates the need to distinguish between $L_{i j}$ and $L_{i j}^{D}$, since $M_{i j}$ is a deviatoric (traceless) tensor in incompressible flows. With planar averaging, this scale-invariant version of dynamic model will henceforth be denoted PASI.

\section{B. The planar-averaged scale-dependent dynamic model}

To account for scale effects in the dynamic model, two approaches exist. If prior knowledge of the variation of $c_{s, \Delta}$ with scale is available, the parameter $\beta$ can be prescribed $a$ priori. Such a "semi-dynamic" approach with an imposed parameter $\beta$ was tested by Meneveau and Lund ${ }^{35}$ to capture scale dependence in the transition from LES to direct numerical simulation (DNS) $(\Delta \rightarrow$ the Kolmogorov scale $\eta$ ) for finely resolved LES. The approach was also used in simulations by Bou-Zeid et al. ${ }^{36}$ for wall-bounded flows in the limit where $\Delta$ tends to the local integral scale near the wall. In both studies, the semi-dynamic scale-dependent model was shown to give better results than the scale-invariant model.

The other option is to implement a fully dynamic formulation where the scale-dependence parameter $\beta$ is measured through an additional filtering operation. The latter approach has been successfully implemented with the planar-averaged dynamic approach for atmospheric boundary-layer (ABL) flows by Porté-Agel et al. ${ }^{22}$ The main assumption used in this model is that a power-law behavior describes the scale dependence of the coefficient, i.e., $c_{s, \Delta} \sim \Delta^{\phi}$ or, in a dimensionally more appropriate form, $c_{s, \Delta}=c_{s, \alpha \Delta}(\Delta / \alpha \Delta)^{\phi}$. As a consequence, $\beta$ evaluated as the ratio of coefficients at scales 
$\alpha \Delta$ and $\Delta$ is equal to $\beta$ evaluated between scales $\alpha^{2} \Delta$ and $\alpha \Delta$ (i.e., the power-law assumption is equivalent to the assumption that the scale-dependence parameter $\beta=\alpha^{2 \phi}$ is itself scale-invariant-see discussion in Porté-Agel et $a .^{22}$ ). A second Germano identity, written between scales $\Delta$ and $\alpha^{2} \Delta$ yields

$$
c_{s, \Delta}^{2}=\frac{\left\langle Q_{i j} N_{i j}\right\rangle}{\left\langle N_{i j} N_{i j}\right\rangle},
$$

where $Q_{i j}$ (the resolved stress tensor between $\Delta$ and $\alpha^{2} \Delta$ ) and $N_{i j}$ are given by

$$
Q_{i j}=\widetilde{\tilde{u}_{i} \widetilde{u}_{j}}-\hat{\hat{u}_{i}} \hat{\tilde{u}}_{j}, \quad N_{i j}=2 \Delta^{2}\left[\widetilde{\widetilde{S} \mid \widetilde{S}_{i j}}-\alpha^{4} \beta^{2}|\hat{\bar{S}}| \hat{\hat{S}_{i j}}\right]
$$

By equating the right-hand sides of Eqs. (7) and (8), Porté-Agel et al. ${ }^{22}$ obtained a fifth-order polynomial in $\beta$, including ten terms (tensor contractions involving various filtered strain-rates and resolved stress tensors) that need to be averaged over directions of statistical homogeneity. ${ }^{22}$ The polynomial is solved for $\beta$ and the solution is then replaced in Eq. (7) to obtain a scale-dependent estimate of $c_{s, \Delta}$. This implementation of the scale-dependent model, used in PortéAgel et al., ${ }^{22}$ worked well with the planar averaging approach.

\section{The Lagrangian-averaged scale-invariant SGS model}

The requirement for homogeneous directions in the flow field limits the use of the dynamic model to relatively simple flows, excluding many practical flows in complex geometries. Local formulations of the model have been developed. ${ }^{25,37}$ As outlined in Sec. I, the need to evaluate the divergence of the SGS stress makes the highly intermittent coefficient fields, which often need to be clipped at zero, resulting from purely local dynamic determinations undesirable. Moreover, conceptually some averaging is needed to recover the statistical basis of the eddy-viscosity model. ${ }^{21,24}$ An alternative approach, combining features from the local and averaged formulations, was developed by Meneveau $e t$ $a l .{ }^{24}$ The model averages the Smagorinsky coefficient in time following fluid pathlines and hence it is called the Lagrangian-averaged scale-invariant model (LASI). The Lagrangian averaging enforces to some degree the statistical basis that supports the use of an eddy-diffusion model and is physically justifiable since turbulent eddies with sizes about the grid scale are likely to be convected along fluid pathlines. Meneveau and Lund ${ }^{38}$ also showed that the turbulent energy cascade is most apparent when viewed in a Lagrangian frame of reference. The model is very well suited for the applications with heterogeneous spatial conditions since it preserves local variability, preserves Galilean invariance, and does not require homogeneous directions. It has already been applied in LES of flows in complex domains such as flows in internal combustion engines, ${ }^{27}$ flow over wavy walls, ${ }^{28}$ flows in thrust reversers, ${ }^{29}$ and flow of impinging jets. ${ }^{30}$
In the Lagrangian SGS model, the coefficient $c_{s, \Delta}$ is obtained by minimizing the weighted time average of the local error contraction $e_{i j} e_{i j}$ over pathlines; this weighted time average can be written as

$$
E=\int_{-\infty}^{t} e_{i j}\left[\mathbf{z}\left(t^{\prime}\right), t^{\prime}\right] e_{i j}\left[\mathbf{z}\left(t^{\prime}\right), t^{\prime}\right] W\left(t-t^{\prime}\right) d t^{\prime},
$$

where $\mathbf{z}\left(t^{\prime}\right)$ are the previous positions of the fluid elements and $W(\tau)$ is a relaxation function that typically allocates larger weights to the more recent history of the coefficients (i.e., $W(\tau)$ is a decreasing function of $\tau$ ). By filtering between $\Delta$ and $\alpha \Delta$ and using a scale invariant form of the Germano identity, the coefficient is obtained by setting the variation of $E$ with respect to $c_{s, \Delta}^{2}$ to zero:

$$
\frac{\partial E}{\partial c_{s, \Delta}^{2}}=\int_{-\infty}^{t} 2 e_{i j} \frac{\partial e_{i j}}{\partial c_{s, \Delta}^{2}} W\left(t-t^{\prime}\right) d t^{\prime}=0,
$$

which results in the following expression for $c_{s, \Delta}^{2}$ :

$$
c_{s, \Delta}^{2}=\frac{\mathcal{J}_{L M}}{\mathcal{J}_{M M}},
$$

where

$$
\mathcal{J}_{L M}=\int_{-\infty}^{t} L_{i j} M_{i j}\left[\mathbf{z}\left(t^{\prime}\right), t^{\prime}\right] W\left(t-t^{\prime}\right) d t^{\prime}
$$

and

$$
\mathcal{J}_{M M}=\int_{-\infty}^{t} M_{i j} M_{i j}\left[\mathbf{z}\left(t^{\prime}\right), t^{\prime}\right] W\left(t-t^{\prime}\right) d t^{\prime} .
$$

For the weighting function, a choice of an exponential form, $W\left(t-t^{\prime}\right)=(1 / T) e^{-\left(t-t^{\prime}\right) / T}$, allows replacing cumbersome evaluations of backward time integrals with forward relaxation-transport equations. Based on DNS results and dimensional self-consistency, ${ }^{24}$ the time scale $T$ is chosen as $T=1.5 \Delta\left(\mathcal{J}_{L M} \mathcal{J}_{M M}\right)^{-1 / 8}$. This choice of the time scale offers the practical advantage of allocating less weight to recent history (i.e., increasing the model's memory) if the current values of $L_{i j} M_{i j}$ are negative. The time scale is effectively infinite if $\mathcal{J}_{L M}$ reaches zero, thus preventing negative values of $c_{s, \Delta}$. The relaxation transport equations thus obtained for $\mathcal{J}_{L M}$ and $\mathcal{J}_{M M}$ are

$$
\frac{D \mathcal{J}_{L M}}{D t}=\frac{\partial \mathcal{J}_{L M}}{\partial t}+\widetilde{\mathbf{u}} \cdot \nabla \mathcal{J}_{L M}=\frac{1}{T_{\Delta}}\left(L_{i j} M_{i j}-\mathcal{J}_{L M}\right)
$$

and

$$
\frac{D \mathcal{J}_{M M}}{D t}=\frac{\partial \mathcal{J}_{M M}}{\partial t}+\widetilde{\mathbf{u}} \cdot \nabla \mathcal{J}_{M M}=\frac{1}{T_{\Delta}}\left(M_{i j} M_{i j}-\mathcal{J}_{M M}\right) .
$$

Using first-order numerical approximations in space and time, these equations can be discretized and included rather economically in an LES code. The resultant formulation to update from time-step " $n$ " to " $n+1$ " at a grid point located at $\mathbf{x}$ is 


$$
\begin{aligned}
& \mathcal{J}_{M M}^{n+1}(\mathbf{x})=\varepsilon\left[M_{i j} M_{i j}\right]^{n+1}(\mathbf{x})+(1-\varepsilon) \mathcal{J}_{M M}^{n}\left(\mathbf{x}-\widetilde{\mathbf{u}}^{n} \Delta t\right), \\
& \mathcal{J}_{L M}^{n+1}(\mathbf{x})=H\left\{\varepsilon\left[L_{i j} M_{i j}\right]^{n+1}(\mathbf{x})+(1-\varepsilon) \mathcal{J}_{L M}^{n}\left(\mathbf{x}-\widetilde{\mathbf{u}}^{n} \Delta t\right)\right\},
\end{aligned}
$$

where

$$
\varepsilon=\frac{\Delta t / T^{n}}{1+\Delta t / T^{n}}, \quad T^{n}=1.5 \Delta\left(\mathcal{J}_{L M}^{n} \mathcal{J}_{M M}^{n}\right)^{-1 / 8}
$$

and

$$
H\{x\}=\text { ramp function }=\mid \begin{array}{cc}
x & \text { if } x \geqslant 0 \\
10^{-32} & \text { otherwise }
\end{array} .
$$

Here $\Delta t$ is the time step. Bilinear spatial interpolation is used to evaluate the previous values at position $\mathbf{x}-\widetilde{\mathbf{u}}^{n} \Delta t$, i.e., "upstream" of the grid point in question. The ramp function is needed to ensure that numerical inaccuracies (mainly due to the discretization of the equations) do not yield slightly negative $c_{s, \Delta}$ values despite the choice of the time scale to avoid such occurrences, and to avoid infinities when evaluating $T^{n}$. For wall-bounded flow simulations (such as LES presented later in this study), periodic boundary conditions for $\mathcal{J}_{L M}$ and $\mathcal{J}_{M M}$ are used in the horizontal directions. At the lower and upper boundaries, zero-gradient (homogeneous Neumann) boundary conditions are imposed, i.e., the values at the boundary are set equal to the values at the closest node inside the domain.

\section{SCALE-DEPENDENT LAGRANGIAN DYNAMIC MODEL}

Implementation of the Lagrangian averaging approach described in Sec. II C with the scale-dependent method described in Sec. II B would require accumulating ten different Lagrangian averages (see Sec. II B and Porté-Agel et $a .^{22}$ ), as well as solving a fifth-order polynomial for $\beta$ at every grid point in the domain. This is a prohibitively expensive procedure, and the choice of the proper polynomial root, if it exists, is difficult due to the more noisy characteristics of Lagrangian averaging compared to planar averaging. Hence, a simplified procedure that is better suited for Lagrangian averaging is sought here

The procedure is based on the observation that the dynamic model, in its scale-invariant formulation [i.e., using $\beta=1$ when evaluating $M_{i j}$ (Eq. (6)], yields a coefficient that corresponds to the test-filter scale $\alpha \Delta$ rather the grid-filter scale $\Delta$. That is to say, in practice one approximately obtains a value suitable for $c_{s, \alpha \Delta}$ instead of $c_{s, \Delta}$ when evaluating the right-hand side of Eqs. (7) or (12) with $M_{i j}$ using $\beta=1$. This effect has been observed to be true in numerical experiments of scale dependence both when the coefficient increases with increasing scale, such as when $\Delta$ approaches the Kolmogorov scale, ${ }^{35}$ and when the coefficient decreases with increasing scale, e.g., when $\Delta$ is close to the integral scale. ${ }^{22}$ This effect can be traced to the fact that, in the Germano identity [Eq. (3)], the tensor terms relating to the test-filter scale $\alpha \Delta$ are significantly greater than the terms relating to the grid-filter scale $\Delta$ (about 4 times greater if $\alpha=2$ ). Hence these terms relating to the test-filter scale dominate the error term [Eq. (5)]. Using this observation and using $\alpha=2$, we may write

$$
c_{s, 2 \Delta}^{2}=\frac{\mathcal{J}_{L M}}{\mathcal{J}_{M M}} .
$$

Similarly, for $\alpha=4$, we write

$$
c_{s, 4 \Delta}^{2}=\frac{\mathcal{J}_{Q N}}{\mathcal{J}_{N N}},
$$

where

$$
\mathcal{J}_{Q N}=\int_{-\infty}^{t} Q_{i j} N_{i j}\left[\mathbf{z}\left(t^{\prime}\right), t^{\prime}\right] W\left(t-t^{\prime}\right) d t^{\prime}
$$

and

$$
\mathcal{J}_{N N}=\int_{-\infty}^{t} N_{i j} N_{i j}\left[\mathbf{z}\left(t^{\prime}\right), t^{\prime}\right] W\left(t-t^{\prime}\right) d t^{\prime} .
$$

Here $Q_{i j}$ and $N_{i j}$ are computed using their definitions given in Eq. (9) and a value of $\beta=1$; however, the weighting function now involves exponential decay with a time constant,

$$
T_{4 \Delta}^{n}=1.5 \Delta\left(\mathcal{J}_{Q N}^{n} \mathcal{J}_{N N}^{n}\right)^{-1 / 8} .
$$

In the Lagrangian scale-dependent model the averages $\mathcal{I}_{Q N}$ and $\mathcal{I}_{N N}$ are evaluated from two additional relaxation transport equations:

$$
\frac{\partial \mathcal{J}_{Q N}}{\partial t}+\widetilde{\mathbf{u}} \cdot \nabla \mathcal{J}_{Q N}=\frac{1}{T_{4 \Delta}}\left(Q_{i j} N_{i j}-\mathcal{J}_{Q N}\right)
$$

and

$$
\frac{\partial \mathcal{J}_{N N}}{\partial t}+\widetilde{\mathbf{u}} \cdot \nabla \mathcal{J}_{N N}=\frac{1}{T_{4 \Delta}}\left(N_{i j} N_{i j}-\mathcal{J}_{N N}\right) .
$$

When they are discretized according to the same procedures and approximations described in the preceding section, they read

$$
\begin{aligned}
\mathcal{J}_{N N}^{n+1}(\mathbf{x})= & \varepsilon_{4 \Delta}\left[N_{i j} N_{i j}\right]^{n+1}(\mathbf{x})+\left(1-\varepsilon_{4 \Delta}\right) \mathcal{J}_{N N}^{n}\left(\mathbf{x}-\widetilde{\mathbf{u}}^{n} \Delta t\right), \\
\mathcal{J}_{Q N}^{n+1}(\mathbf{x})= & H\left\{\varepsilon_{4 \Delta}\left[Q_{i j} N_{i j}\right]^{n+1}(\mathbf{x})+\left(1-\varepsilon_{4 \Delta}\right)\right. \\
& \left.\times \mathcal{J}_{Q N}^{n}\left(\mathbf{x}-\widetilde{\mathbf{u}}^{n} \Delta t\right)\right\},
\end{aligned}
$$

where

$$
\varepsilon_{4 \Delta}=\frac{\Delta t / T_{4 \Delta}^{n}}{1+\Delta t / T_{4 \Delta}^{n}}, \quad T_{4 \Delta}^{n}=1.5 \Delta\left(\mathcal{J}_{Q N}^{n} \mathcal{J}_{N N}^{n}\right)^{-1 / 8}
$$

and

$$
H\{\mathrm{x}\}=\text { ramp function }=\mid \begin{array}{cc}
x & \text { if } x \geqslant 0 \\
10^{-32} & \text { otherwise }
\end{array} .
$$

Boundary conditions are similar to those used for $\mathcal{J}_{L M}$ and $\mathcal{J}_{M M}$ (periodic in the horizontal directions and zero gradient at the lower and upper boundaries). In the source terms, both $M_{i j}$ and $N_{i j}$ are evaluated assuming that $\beta=1$ (which can be considered as a "first guess" in an iterative, explicit 
procedure to take scale-dependence into account). From the locally known approximate values of $c_{s, 2 \Delta}$ and $c_{s, 4 \Delta}$, the local value of $\beta$ can be evaluated according to

$$
\beta=c_{s, 4 \Delta}^{2} / c_{s, 2 \Delta}^{2} \text {. }
$$

Next, as in Porté-Agel et al., ${ }^{22}$ we use the assumption that $\beta$ is scale invariant, i.e., $\beta=c_{s, 4 \Delta}^{2} / c_{s, 2 \Delta}^{2}=c_{s, 2 \Delta}^{2} / c_{s, \Delta}^{2}$ (which amounts to postulating power-law dependence of the coefficient as a function of scale, with arbitrary exponent). We may then solve for the unknown coefficient at scale $\Delta$,

$$
c_{s, \Delta}^{2}=c_{s, 2 \Delta}^{2} / \beta,
$$

where $\beta$ is given by Eq. (26).

In principle, $\beta$ obtained from Eq. (26) can vary between 0 and infinity, depending on the local values obtained for $c_{s, 4 \Delta}$ and $c_{s, 2 \Delta}$. In practice, of course, some of these limits may cause numerical difficulties. We have observed that in the limit of $\beta \rightarrow \infty$ (i.e., when $c_{s, 2 \Delta}$ tends to zero while $c_{s, 4 \Delta}$ does not), the local coefficient used in LES goes to zero (smoothly) and this limit does not pose any difficulties in LES. However, when $c_{s, 4 \Delta}$ tends to zero while $c_{s, 2 \Delta}$ does not, $\beta \rightarrow 0$, leading to very large values of $c_{s, \Delta}$ from Eq. (27). In simulations, this may lead to numerical instabilities associated with viscous stability conditions being locally violated. Therefore, some clipping of $\beta$ away from zero is required to allow simulations to proceed. We choose a lower limit of $\beta$ $\geqslant 0.125$. The clipping limit is significantly below physically expected limiting behaviors. As the ground is approached, the mixing length $\gamma=\Delta c_{s, \Delta}$ is expected to become proportional to the integral scale $z$. Therefore, close to the ground $\Delta c_{s, \Delta} \sim z$ or $c_{s, \Delta} \sim z / \Delta$. Therefore, as the wall is approached, the average value of $\beta$ tends to

$$
\beta=c_{s, 2 \Delta}^{2} / c_{s, \Delta}^{2} \sim \frac{z^{2} /(2 \Delta)^{2}}{z^{2} / \Delta^{2}}=1 / 4 .
$$

Away from the wall, $\beta$ is expected to increase to a mean value of about 1 . Hence, the clipping limit of 0.125 allows the coefficient to decrease locally to half its average minimum value. Note that this clipping limit already corresponds to very severe scale dependence, namely, the eddy-viscosity coefficient at scale $\Delta$ being eight times larger than at scale $2 \Delta$.

No limit is imposed for high $\beta$, thus the allowable range is $0.125 \leqslant \beta \leqslant \infty$. Tests with other clipping limits were conducted ( $\beta$ was clipped at 0.1 and 0.167 ) without significant impact on the results. This was expected since it is observed in simulations that the clipping at 0.125 is only needed about $15 \%$ of the time: the clipping limit cannot significantly affect the results or be used as a tuning parameter to adjust the results. Therefore, the model coefficient to be used in the simulation is obtained at every grid point and time step from the Lagrangian averaged quantities according to

$$
c_{s, \Delta}^{2}=\frac{c_{s, 2 \Delta}^{2}}{\max (\beta, 0.125)}=\frac{\mathcal{J}_{L M} / \mathcal{J}_{M M}}{\max \left(\frac{\mathcal{J}_{Q N} \mathcal{J}_{M M}}{\mathcal{J}_{N N} \mathcal{J}_{L M}}, 0.125\right)} .
$$

In summary, the Lagrangian-averaged scale-dependent dynamic model (LASD) involves test-filtering and second test-filtering operations to evaluate the local source terms $L_{i j} M_{i j}, M_{i j} M_{i j}, Q_{i j} N_{i j}$, and $N_{i j} N_{i j}$, updating the four Lagrangian averages $\mathcal{J}_{L M}, \mathcal{J}_{M M}, \mathcal{J}_{Q N}$, and $\mathcal{J}_{N N}$ following Eqs. (17) and (25), and evaluating the local coefficient value to be used in LES according to Eq. (29).

The same scale-dependent approach can obviously be used with planar averaging where the planar averaged coefficient at the grid-filter scale can be obtained using

$$
c_{s, \Delta}^{2}=\frac{\left\langle L_{i j} M_{i j}\right\rangle /\left\langle M_{i j} M_{i j}\right\rangle}{\left(\frac{\left\langle Q_{i j} N_{i j}\right\rangle\left\langle M_{i j} M_{i j}\right\rangle}{\left\langle N_{i j} N_{i j}\right\rangle\left\langle L_{i j} M_{i j}\right\rangle}\right)} .
$$

The brackets denote planar averaging. This planar-averaged scale-dependent model will be referred to as PASD. No clipping is needed with the planar averaged approach since the heavy averaging associated with the planar formulation eliminates the fluctuations that led to the need for clipping.

\section{NUMERICAL CODE AND TESTS IN HOMOGENEOUS, HIGH-REYNOLDS NUMBER, BOUNDARY LAYER FLOWS}

\section{A. Numerical code}

The isothermal LES equations are solved in rotational form to ensure conservation of mass and kinetic energy of the inertial terms: ${ }^{39}$

$\frac{\partial \widetilde{u}_{i}}{\partial x_{i}}=0$

$\frac{\partial \widetilde{u}_{i}}{\partial t}+\tilde{u}_{j}\left(\frac{\partial \tilde{u}_{i}}{\partial x_{j}}-\frac{\partial \widetilde{u}_{j}}{\partial x_{i}}\right)=-\frac{1}{\rho} \frac{\partial \tilde{p}^{*}}{\partial x_{i}}+\frac{\partial}{\partial x_{j}}\left(2\left(c_{s, \Delta} \Delta\right)^{2}|\widetilde{S}| \tilde{S}_{i j}\right)+\widetilde{F}_{i}$.

Here $F_{i}$ is the mean streamwise pressure forcing. Note that in the above equations, the molecular viscous term is neglected because the paper focuses on very high-Reynolds number flows where viscosity is negligible at the resolved scales and the wall layer is modeled (as opposed to resolving the viscous sublayer, see Pope ${ }^{26}$ ). The modified pressure term

$$
\widetilde{p}^{*}=\widetilde{p}+(1 / 3) \rho \sigma_{k k}+(1 / 2) \rho \widetilde{u}_{j} \widetilde{u}_{j}
$$

is computed as usual from a Poisson equation (divergence of momentum equation set to zero due to continuity condition).

The code uses a pseudospectral approach in the horizontal directions. A second-order accurate centered-differences scheme, requiring a staggered grid, is used in the vertical direction. This entails storing the variables at heights $j d z$ or $(j+1 / 2) d z$; where $j$ goes from 0 to $N$ (the number of vertical grid points). The fully explicit second-order accurate AdamsBashforth scheme is used for time advancement. Aliasing errors can be detrimental to the accuracy of the SGS parametrization since they affect the smallest resolved scales used to compute the dynamic Smagorinsky coefficient. To overcome this problem, the $3 / 2$ rule $^{40}$ is used to fully dealias the convective terms. More details about some numerical aspects of the code (unrelated to the SGS model) can be found in Refs. 7 and 8. 
TABLE I. Simulation parameters for homogeneous surface simulations.

Domain height

Domain length $L$ and width $W$

Mesh spacings

Wall roughness $z_{0}$ imposed in lower boundary condition

Number of grid points

Initial conditions

Warm-up period

Forcing

Simulation time step, $d t$

Lagrangian model time step

Number of simulation time steps

Output sampling frequency
$H$

$L=W=2 \pi H$

$d x=d y=2 \pi d z$

$z_{0} / H=10^{-4}$

$N^{3}=64^{3}$

Mean velocity: logarithmic profile near the surface merging smoothly with a zero-gradient profile at the top. Velocity fluctuations: imposed randomly (in space and among components) on the mean profile using a prescribed turbulent kinetic energy profile (from results by Andren et al. ${ }^{\mathrm{a}}$ ).

Warm-up simulations are run until the normalized total stress profile adjusts to a straight line reaching 1 at the surface and the mean resolved kinetic energy is stable.

Imposed pressure gradient: $(1 / \rho) \boldsymbol{\nabla} p=u_{*}{ }^{2} / H$.

$d t u_{*} / H=0.00025$ (nondimensional time units).

$\Delta t=5 d t$

200000

Every ten time steps

${ }^{\mathrm{a}}$ Reference 43 .

The boundary conditions in the horizontal directions are periodic. A stress free condition is imposed at the top of the domain by setting

$$
\partial_{3} \tilde{u}_{1,2}=\tilde{u}_{3}=0
$$

where 1,2 , and 3 (or $x, y, z$ in other parts of the paper) refer to the streamwise, cross-stream, and vertical directions, respectively. At the bottom of the domain, the vertical velocity is set to 0 at the surface. As a consequence of the staggered grid formulation, no boundary conditions are needed for the horizontal velocities since they are only stored at a distance $d z / 2$ above the surface. Stresses at the surface are imposed through a local similarity theory formulation ${ }^{41}$ (see Piomelli and Balaras ${ }^{42}$ for a review of wall modeling in LES). However, velocities filtered at twice the grid scale are used to compute the surface stress; this is needed to ensure that the average stress over the wall is close to the stress predicted by the classic log law. The need for this formulation and its derivation are explained in the Appendix. The resulting lawof-the-wall formulation is

$$
\begin{aligned}
\tau_{w}(x, y)= & -\left[\frac{\kappa}{\ln \left[(d z / 2) / z_{o}\right]}\right]^{2}\left(\left[\overline{\tilde{u}}_{1}(x, y, d z / 2)\right]^{2}\right. \\
& \left.+\left[\overline{\tilde{u}}_{2}(x, y, d z / 2)\right]^{2}\right) .
\end{aligned}
$$

Subsequently, the stress is partitioned into its streamwise and cross-stream components in the usual manner:

$$
\tau_{i, 3}^{\text {wall }}(x, y)=\tau_{w}(x, y)\left[\frac{\overline{\tilde{u}}_{i}(x, y, d z / 2)}{\sqrt{\overline{\tilde{u}}_{1}^{2}+\overline{\tilde{u}}_{2}^{2}}}\right], \quad i=1,2 .
$$

A sharp spectral cutoff filter is used in the wall stress and SGS computations. The fully scale dependent dynamic model increases the computational cost by about $20 \%$ compared to the Smagorinsky-Lilly model with imposed coefficient. Half of this increase is related to the dynamic computations of the coefficient while the other half is related to the Lagrangian averaging operations. These computational re- quirements are assessed when the coefficient $c_{s, \Delta}$ is updated every fifth time step; this update frequency of the coefficient requires a time step for Lagrangian model $\Delta t[\mathrm{Eq}$. (17) and (25)] equal to five times the time step of the LES code $d t$. Tests for this study confirm that results obtained when updating the coefficient every single time step and every fifth time step are similar.

\section{B. Tests in homogeneous boundary layer flow}

Simulations over a homogeneous rough surface (with constant roughness $z_{o}$ imposed through the stress boundary condition at the lower surface) were performed using: the simple Smagorinsky or Smagorinsky-Lilly model with a wall damping function (SMAG), the PASI, the LASI, the PASD, and the LASD models. Table I details the parameters of the simulations.

The $c_{s, \Delta}$ coefficients computed by the different models (or imposed for the SMAG model) for the homogeneous surface simulations are compared in Fig. 1. Close to the surface, all the dynamic formulations predict a lower value for $c_{s, \Delta}$ than the one assumed by the Mason-Thompson damping function, whereas in the core of the flow they tend towards the classic value between 0.1 and 0.22 . Regardless of the averaging method, the scale-dependent formulation yields larger coefficients than the scale-invariant formulation, consistent with the fact that near the surface the coefficient increases with decreasing scale. In addition, the planar averaged formulations predict lower $c_{s, \Delta}$ than the equivalent Lagrangian formulation away from the wall and a higher $c_{s, \Delta}$ close to the wall. We have verified that the larger Lagrangian averaged mean coefficients occur due to infrequent large values of $c_{s, \Delta}$ obtained locally that dominate the mean values plotted but do not necessarily increase the mean SGS flux or mean dissipation to the same degree. Specifically, results presented later will show that the average dissipation character- 


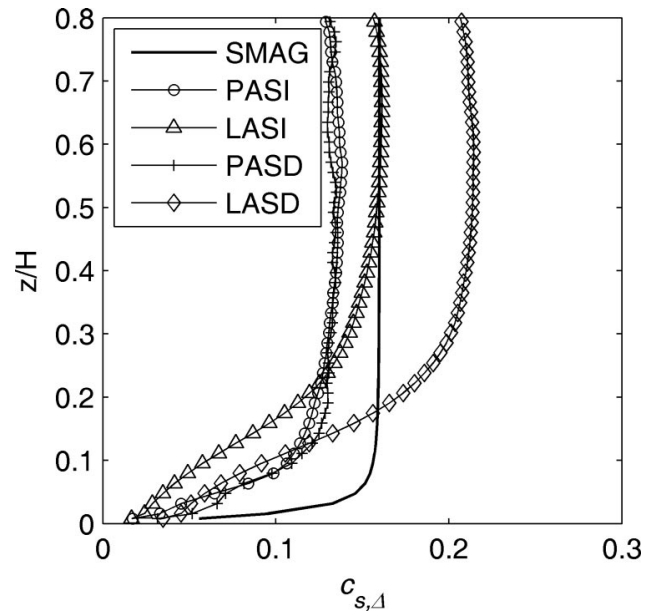

FIG. 1. Vertical profiles of the Smagorinsky coefficient $\left\langle c_{s, \Delta}\right\rangle_{x, y, t}$ for the different SGS eddy-viscosity models.

istics for the different models are not influenced significantly by the averaging method but are much more sensitive to the scale-dependence of the model.

Next, we present mean velocity profiles resulting from the simulations. For the homogeneous surface simulations, the velocity profile is expected to be logarithmic close to the surface (in the bottom 10\%-20\% of the simulation domain), following $\langle\widetilde{u}\rangle=\left(u^{*} / \kappa\right) \ln \left(\mathrm{z} / \mathrm{z}_{\mathrm{o}}\right)$. Figure 2 depicts the mean velocity [Fig. 2(a)] and the nondimensional velocity gradient $\left(\kappa z / u^{*}\right) \partial\langle\widetilde{u}\rangle / \partial z$ [Fig. 2(b)] obtained using the different SGS models. The solid black line in Fig. 2(a) is the log law prediction with $\mathrm{z}_{\mathrm{o}} / H=10^{-4}$. The Smagorinsky model results in a high gradient near the wall. This is in agreement with the previous findings ${ }^{22,32}$ suggesting that the model overdissipates resolved kinetic energy close to solid boundaries and thus the total Reynolds stresses are too low there leading to excessive mean velocity towards the core. Inversely, the two scale-invariant formulations (PASI and LASI) produce insufficient dissipation leading to low velocity gradients and low streamwise velocity near the wall. On the other hand, the scale-dependent formulations yield a value of $\left(\kappa z / u^{*}\right) \partial\langle\tilde{u}\rangle / \partial \mathrm{z}$ close to 1 near the surface suggesting that they are dissipating energy at a more appropriate rate and hence reproducing the log-law region more successfully than the other models. Note also that the present plane averaged scale-dependent results are slightly inferior to those presented previously. ${ }^{22}$ The discrepancy could be due to the fact that, in Ref. 22, a fifth-order polynomial was solved for $\beta$, instead of the more approximate method employed here based on Eq. (30).

Reproducing the log-law region depends on the ability of the SGS model to provide the correct dissipation rate close to the wall. However, a more complete insight into the energy dissipation characteristics of the SGS closure can be obtained by examining the streamwise velocity spectra. In the inertial subrange $\left(k_{1} z>1\right.$, where $k_{1}$ is the wavenumber and $\mathrm{z}$ is the distance to the wall), the effects of viscosity, boundary conditions, and large scale structures are not important and the turbulence is essentially isotropic. The energy cascade in this subrange follows the Kolmogorov spectrum yielding a
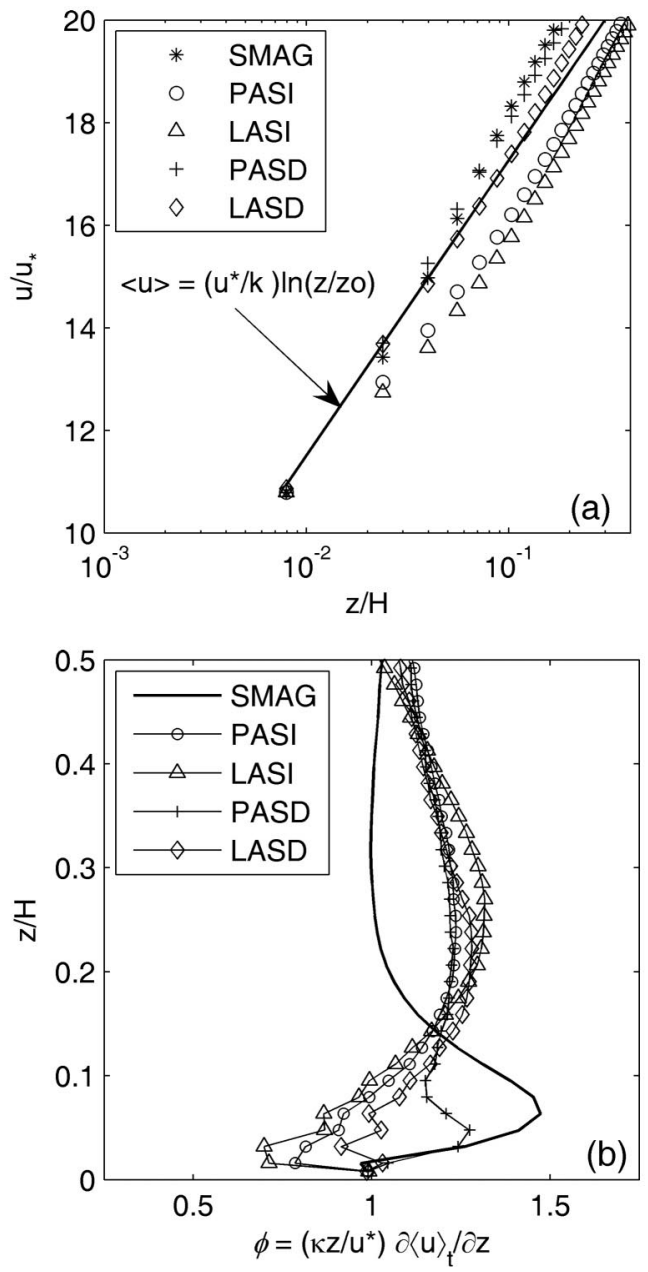

FIG. 2. (a) Normalized streamwise mean velocity profiles; the solid black line is the log law profile with $z_{\mathrm{o}} / H=10^{-4}$, (b) nondimensional mean velocity gradients for different SGS models.

slope of $-5 / 3$. In the production range $\left(k_{1} z<1\right)$, the energy cascade is affected by the flow configuration. In wallbounded flows with neutral stability, there is evidence that the longitudinal (in the streamwise direction) energy spectrum of streamwise velocity in the production range follows a slope of $-1{ }^{44-46}$ We remark that there exists evidence (see, e.g., Refs. 47-49) that the $k^{-1}$ regime does not extend over significant ranges of wavenumbers, and thus the matter is not conclusively settled from the data. Nevertheless, at the relatively short range of scales afforded by the resolution level of our simulations, the expectation of an approximate $k^{-1}$ region still provides a useful criterion to test the various models. Figure 3 depicts the longitudinal $u$ spectra produced by the different SGS models. The conclusions are similar to what was discussed for the log-law prediction. With the Smagorinsky model, too much energy is dissipated and the spectra decay much too fast at high wavenumbers. The Lagrangian scale-invariant formulation (LASI) gives spectra that are too flat at small distances from the surface, indicating insufficient energy dissipation and a buildup of energy at the smallest resolved scales. The spectra of the PASI model are not shown here but depict underdissipation problems near the wall very similar to the LASI model results. The spectra for 

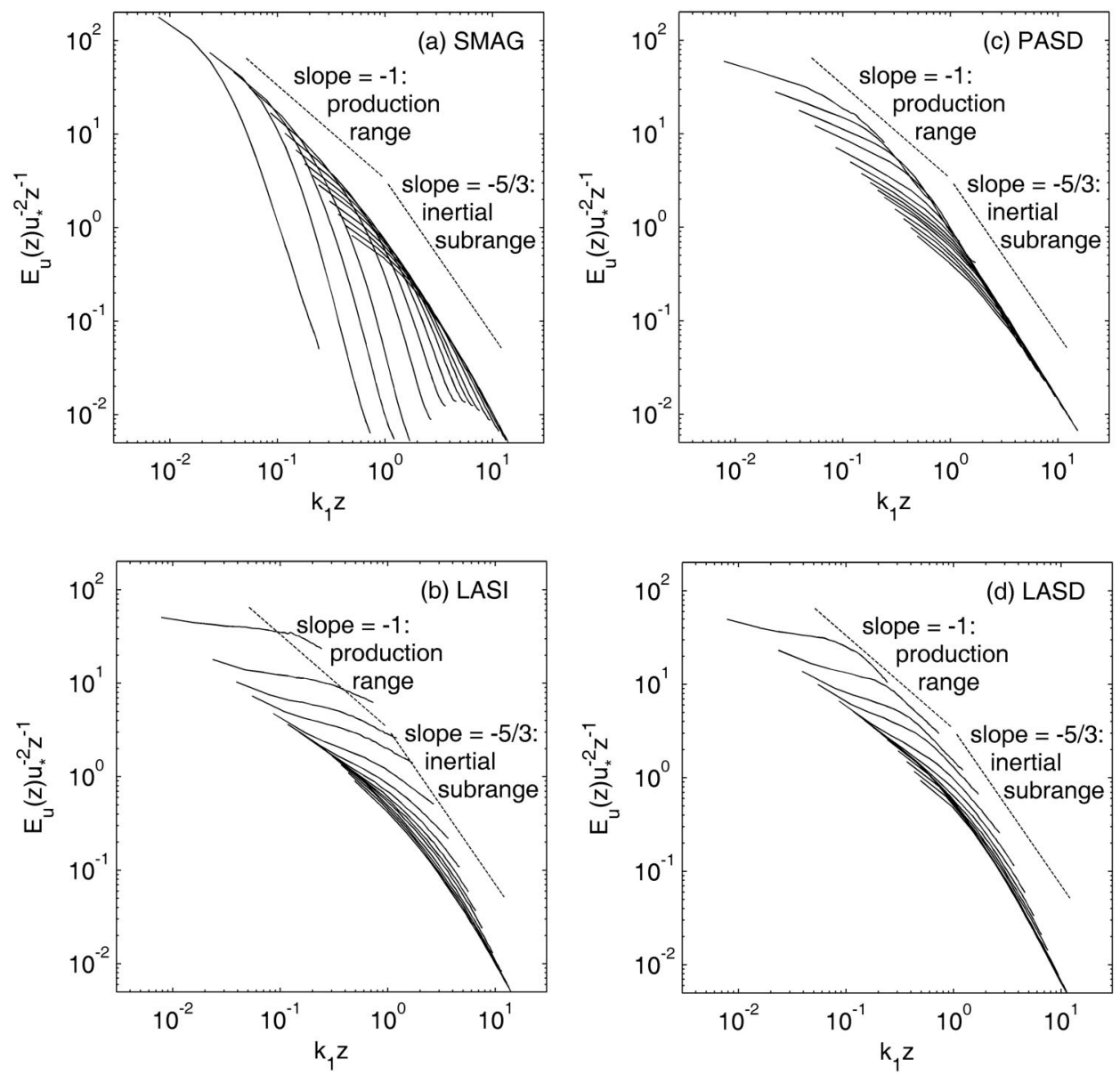

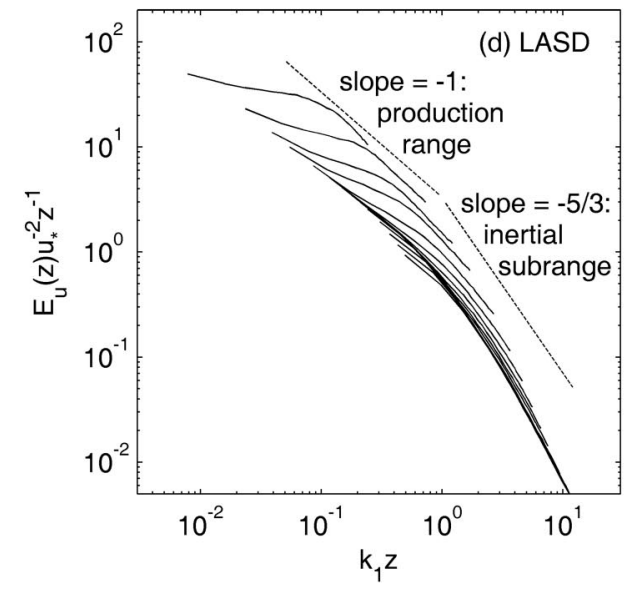

FIG. 3. Normalized streamwise spectra of streamwise velocity vs $k_{1} z$ (the lines represent the spectra obtained at heights $z / H=0.008, \quad 0.024, \quad 0.04$, $0.056,0.087,0.119,0.151,0.183$, $0.214,0.246,0.31,0.373,0.437$, and $0.5)$. the SMAG, LASI, and PASI models are most obviously in error close to the wall, where the assumption of isotropic homogeneous turbulence and the assumption of scale invariance clearly do not hold. The spectra obtained with the LASD and PASD models follow the -1 and $-5 / 3$ slopes well in the two ranges. This confirms that a dynamic scaledependent formulation is important for non wall-resolving LES in the vicinity of walls. Notice also that the LASD model is slightly less dissipative than the PASD model. The same is true when comparing the scale-invariant versions of the planar-averaged and Lagrangian-averaged model (this holds for the results in this study and in Ref. 24).

\section{Lagrangian scale dependent model detailed results}

In the preceding section, basic results of the LASD model were presented and compared with other SGS models. More comprehensive results obtained with the LASD model are presented in this section along with an analysis of the results sensitivity to the resolution of the simulation. The LES simulations over a homogeneous surface were run at resolutions of $32^{3}, 64^{3}, 96^{3}$, and $128^{3}$.

Figures 4(a) and 4(b) depict the resolved, subgrid-scale, and total stress profiles for the $64^{3}$ and $128^{3}$ resolutions. As expected, close to the surface most of the stress is in the SGS part while away from the wall most of the stress is resolved. The results are in qualitative agreement with many LES studies (see Ref. 22 or the comparative study in Ref. 43, for example). Quantitative comparison is difficult since the partitioning of the stress into resolved and SGS components depends on the resolution and the SGS model used. The effect of resolution can be easily observed in Fig. 4 where the $128^{3}$ resolution results in higher resolved stresses (and lower SGS stresses) compared to the $64^{3}$ simulation. The total stresses remain the same but the higher resolution simulation can resolve a larger part of these stresses.

The variances of the resolved velocities for the different resolutions are plotted in Fig. 5 versus $z / \Delta$. The results of the different resolutions match reasonably well. The profiles collapse at small $z / \Delta$ whereas further up an increase in resolution leads to an increase in the resolved variances. The variances of the current LES results fall well within the range reported by Andren et $a l^{43}$ in their comparative study of four LES codes. In addition, the results coincide well with the variances reported by Porté-Agel et al. ${ }^{22}$ in their LES of ABL flow with a planar scale-dependent dynamic model that uses a different approach to "measure" scale dependence (see Sec. II B).

We confirm that the mean coefficient determined from LASD is, to a good approximation in this case of neutrally buoyant homogeneous boundary layer flow, a universal function of height $z$ when normalized with the filter scale $\Delta$. The vertical profiles of $\left\langle c_{s, \Delta}\right\rangle$ computed at different resolutions are plotted versus $z / \Delta$ in Fig. 6. It is clear that, except for 

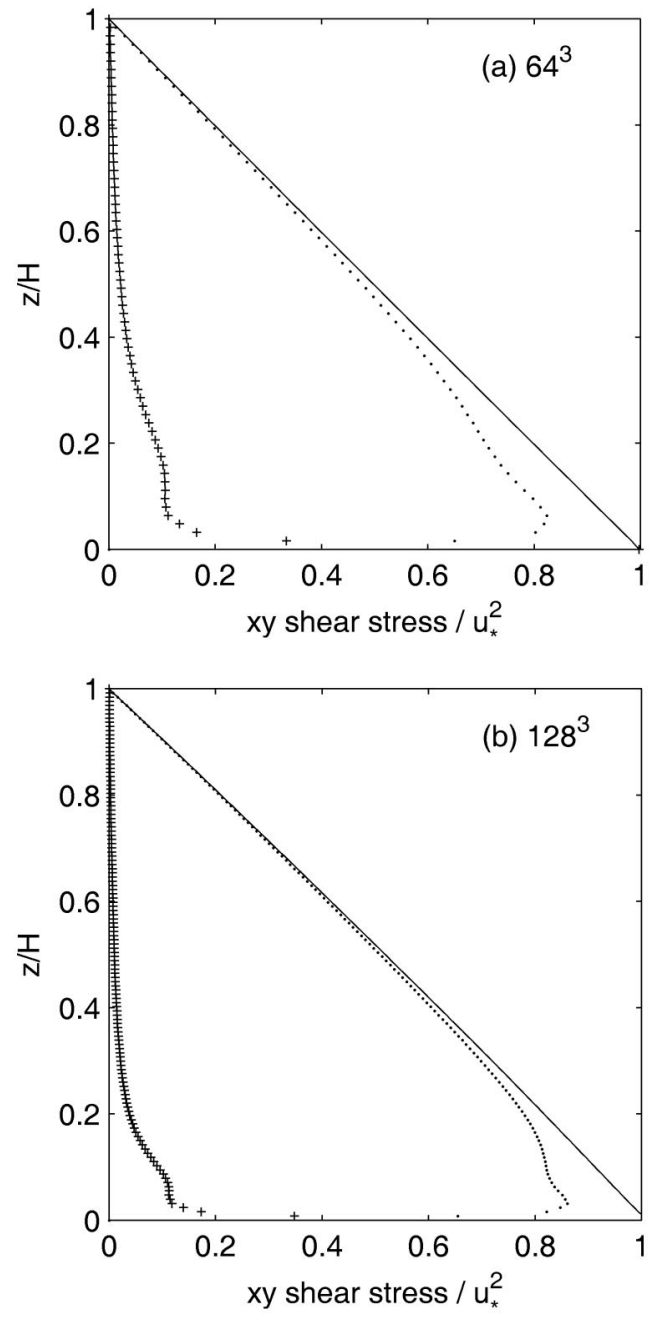

FIG. 4. Vertical profiles of the resolved stress $-\left\langle\widetilde{u}^{\prime} \widetilde{w}^{\prime}\right\rangle_{t, y, x}(\ldots)$, the subgridscale stress $-\left\langle\tau_{x z}\right\rangle_{t, y, x}(+)$, and the total stress (solid line,- -): (a) $64^{3}$ resolution, (b) $128^{3}$ resolution.

small deviations for the $32^{3}$ simulation (due to the effect of the top boundary condition in that case), the results collapse well near the wall.

Next, the variability of the coefficient computed from LASD is documented. Figure 7 depicts the probability den-

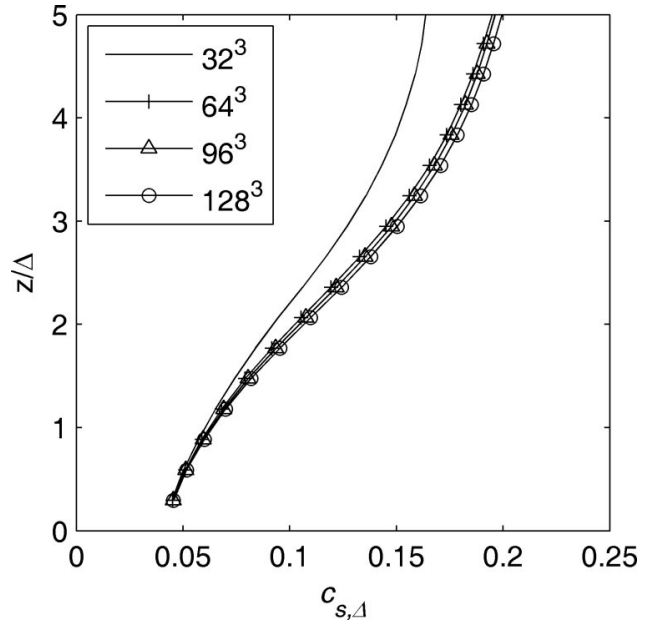

FIG. 6. Collapse of the vertical profiles of the mean dynamic coefficient obtained from LASD model for different resolutions when plotted against $z / \Delta$.

sity functions (pdfs) of the dynamic coefficient at three different heights; for each height, the pdfs obtained from the four different resolutions are shown. The distribution has low standard deviation and mean at $z=d z / 2$, the mean as well as the spread of the data increases further up. Consistent with the pdfs of Lagrangian coefficients determined using the scale-invariant dynamic model in wall-resolving LES, ${ }^{24}$ the peaks of the pdfs are close to the mean values and the pdfs do not exhibit secondary peaks or unusual features. Also note the spike in the pdf at zero associated with the use of a time scale that becomes infinite as $c_{s}$ tends to 0 ; this is in agreement with the scale-invariant version of the model. ${ }^{24}$

For the $32^{3}$ resolution, considerable difference can be noted between the pdfs at $z=H / 8$ and $z=H / 4$. For the higher resolution runs, this difference decreases; this is a direct consequence of the decrease in the ratio of the grid scale $\Delta$ to the integral length scale $\sim \mathrm{z}$, at a given height, as the resolution is increased. Therefore, as the resolution increases, we note a decrease in the height at which the turbulence near the gridscale approaches isotropy.

Figure 8 depicts the probability density function of the scale-dependence parameter $\beta$ and the threshold of $1 / 8$
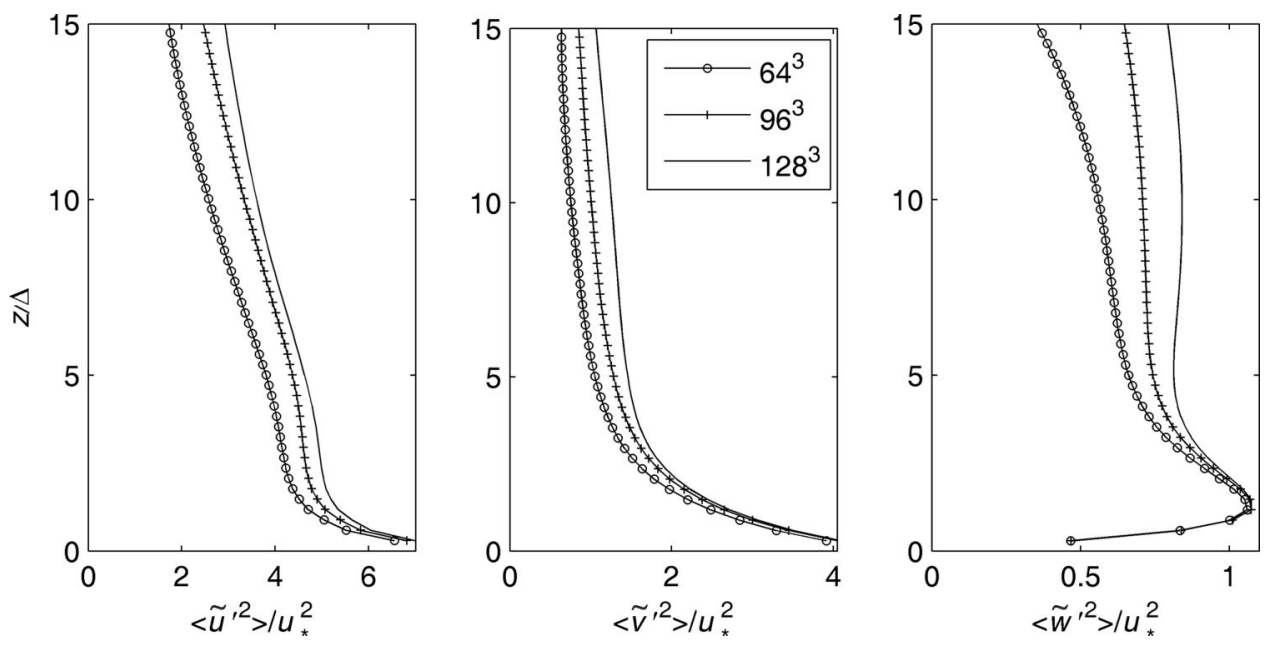

FIG. 5. Normalized variances of resolved velocity components obtained with the LASD model at different resolutions. 

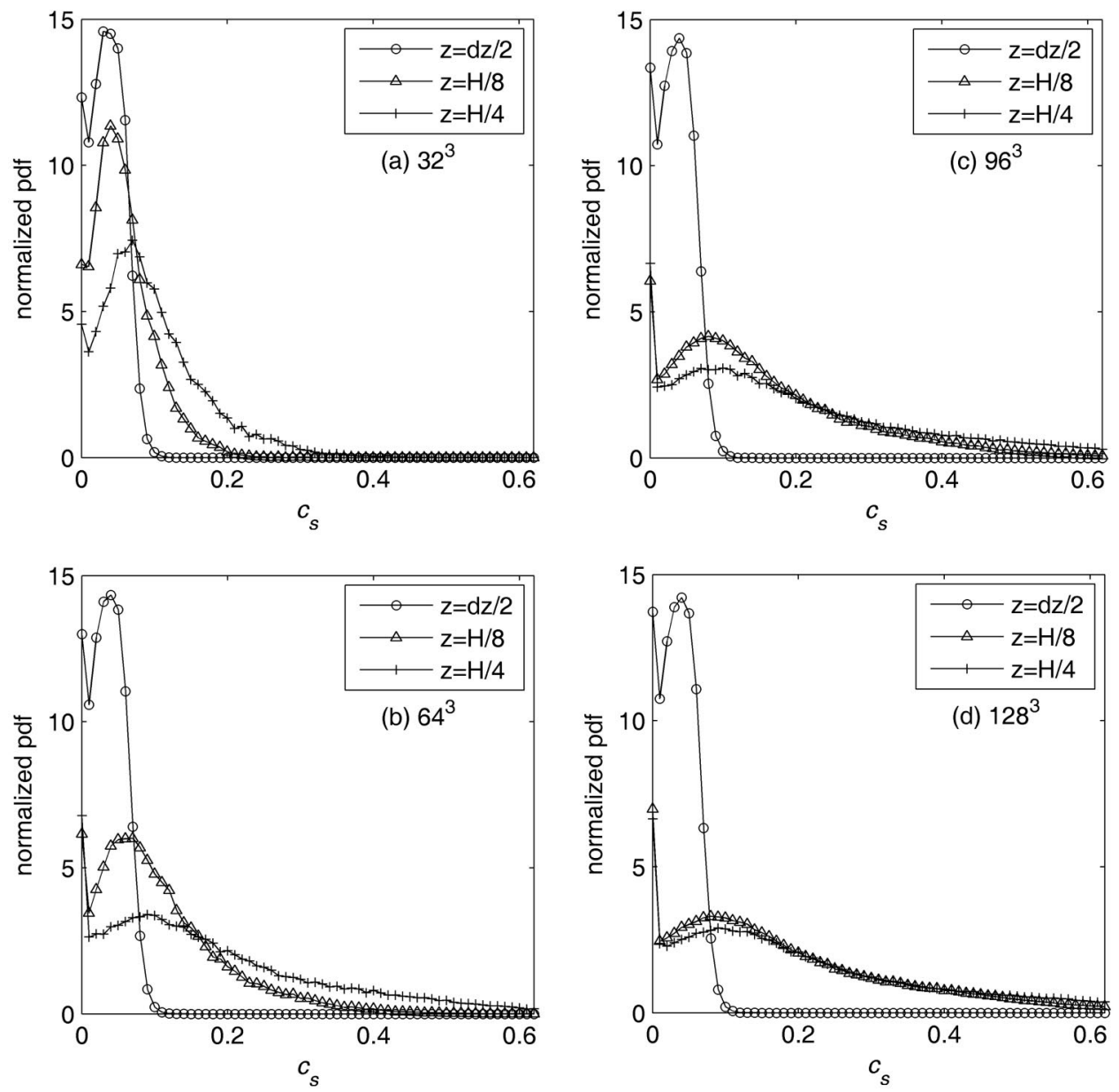

FIG. 7. Probability density functions for the dynamic coefficient $c_{s, \Delta}$ computed using LASD, at different heights and different resolutions: (a) $32^{3}$, (b) $64^{3}$, (c) $96^{3}$, and (d) $128^{3}$. above which it is clipped. Peaks of the pdfs are well above the clipping limit except at $z=d z / 2$ where the peak is close to the clipping limit; however, even at $d z / 2$, most of the $\beta$ values occur above the clipping limit. A small increase in the pdf can be noticed at the value of zero, caused by the use of a Lagrangian model time scale to avoid negative values of $c_{s, 4 \Delta}$, which yields a slightly increased probability of $c_{s, 4 \Delta}$ $=0$ [i.e., $\beta=0$ according to Eq. (26)].

\section{SIMULATIONS OF BOUNDARY LAYER FLOW OVER A HETEROGENEOUS ROUGH SURFACE}

The previous results focused on flow over homogeneous rough surfaces and showed that the scale-dependent approach yields better results than the ad hoc Smagorinsky model or the scale-invariant formulations near the surface. Nevertheless, the motivation for using a Lagrangian local model rather than a plane averaged model becomes apparent only when examining more complex, nonhomogeneous flows (i.e., when homogeneous directions are not available for averaging).

In the simulations over heterogeneous walls presented below, while averaging over horizontal planes is possible, the streamwise direction is not homogeneous and hence the variability of the coefficient in that direction would be suppressed by planar averaging. In such flows, an important question is whether spatial variations in the coefficient are great enough to justify the extra cost of the Lagrangian model (about 10\% more than the equivalent planar averaged version). Moreover, one should consider whether the variations actually affect relevant parameters such as fluid velocities and stresses. These two questions will be addressed in this section where simulations over heterogeneous walls are performed using the LASD and the PASD models and compared to experimental data. The other models (SMAG, LASI, and PASI), having already performed poorly in simulations over homogeneous surfaces, will not be tested.

\section{A. Bradley's experimental setup}

The atmospheric boundary layer measurements by Bradley ${ }^{50}$ have often been used to validate theoretical and numerical models for flow over an abrupt change in surface roughness. ${ }^{51-54}$ Bradley measured the surface stress (with drag plates) and the velocity profiles upstream and downstream of a sudden jump in surface roughness. The measurements were performed over a tarmac (surface roughness $z_{\mathrm{o}}$ $=0.002 \mathrm{~cm})$. A patch with a higher roughness $\left(z_{0}=0.25 \mathrm{~cm}\right)$ was created inside the tarmac by laying artificial roughness mats consisting of vertical spikes with reinforcing mesh in between.

For the low-to-high roughness transition measurements, the high-roughness patch was created at the downstream end of the tarmac and measured $26 \mathrm{~m} \times 20 \mathrm{~m}$ in the streamwise and cross-stream directions, respectively. The tarmac had an effective area upstream of the measurement of 



FIG. 8. Probability density functions of the scale-dependence parameter $\beta$ obtained dynamically from the LASD
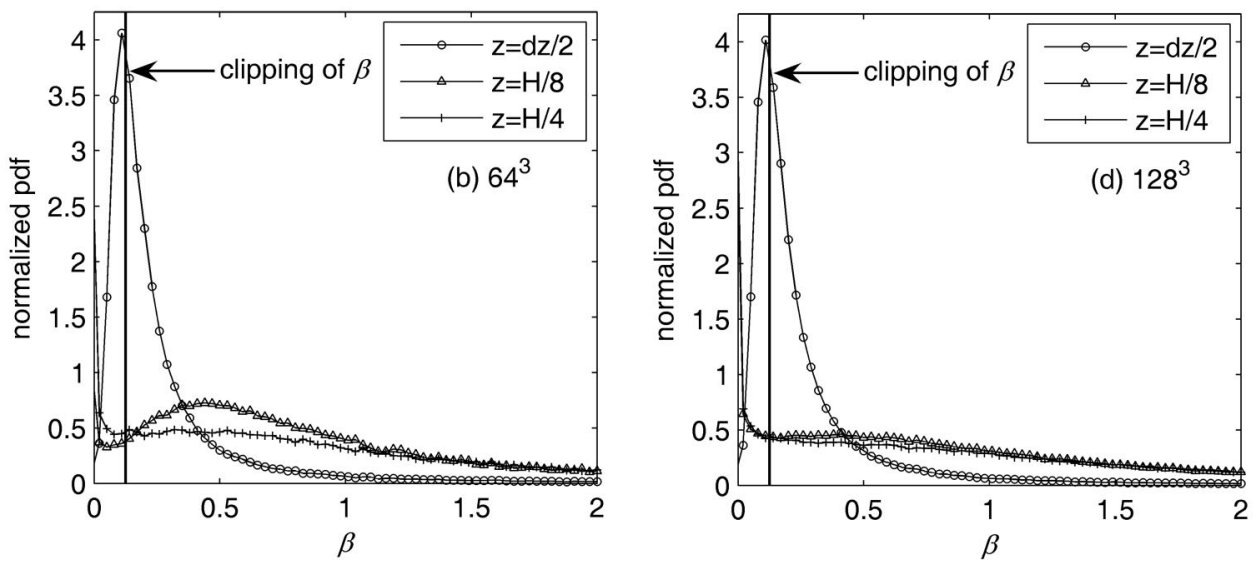
approach, at different heights and different resolutions: (a) $32^{3}$, (b) $64^{3}$, (c) $96^{3}$, and (d) $128^{3}$.

$134 \mathrm{~m} \times 92 \mathrm{~m}$ in the streamwise and cross-stream directions, respectively. For the high-to-low roughness measurements, the high-roughness patch was slightly shorter, measuring $22 \mathrm{~m}$ in the streamwise direction, and the patch was placed near the upstream end of the tarmac.

In the LES, in the cross-stream direction, a domain width of 64 meters is simulated with a resolution $d y=1 \mathrm{~m}$. Furthermore, the high-roughness patch is assumed to extend over the entire cross-stream direction of the simulation (i.e., infinitely wide patch). For the results examined in this work, the cross-stream dimensions of the tarmac and the highroughness patch have very little impact on the results; this was confirmed in LES tests not presented in this work that included rectangular patches of various widths. The computational domain height is $H=20$ meters, i.e., only the nearsurface layer of the ABL is simulated to allow for a high vertical resolution $(d z=10 \mathrm{~cm})$ that can capture internal boundary layers originating at the transition between patches of different roughness. Figure 9 depicts the computational domain used to simulate conditions similar to Bradley's field experiments. Two simulations were performed to reproduce Bradley's low-to-high and high-to-low roughness transitions.

Note that, due to the pseudospectral approach used in the code, what is actually being modeled is an infinite sequence of high-roughness and low-roughness (tarmac) patches. This does not affect the comparison results since we look at stress and velocity at the roughness jump, very close to the wall where the upstream conditions should not be very important. In practice, since the upstream conditions outside of the tarmac are unknown for the Bradley experiment, no better alternative to define inflow conditions exist.

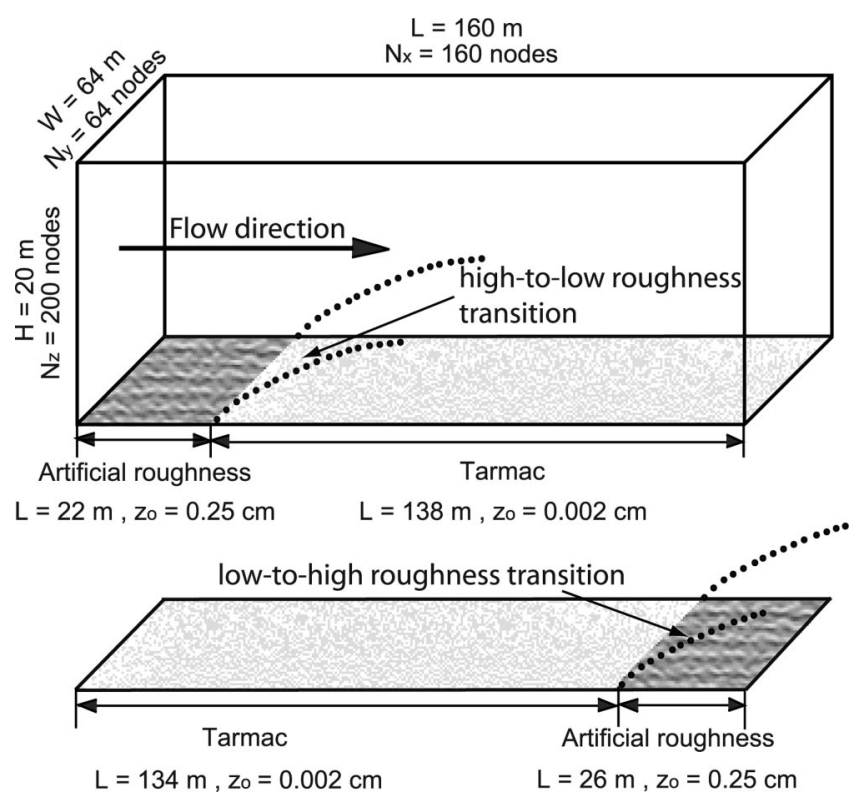

FIG. 9. LES parameters and simulation domain for reproducing Bradley's field experimental study of atmospheric surface layer flow over abrupt roughness transitions. 


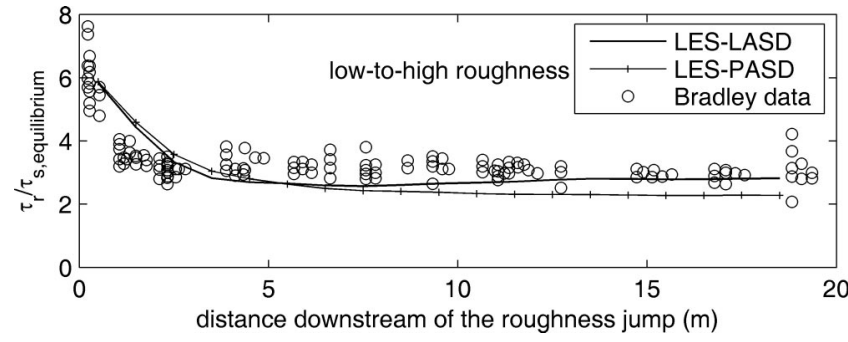

FIG. 10. Wall shear stress downstream of a low-to-high roughness jump, normalized by the equilibrium stress of the upstream surface: comparison of LES results and Bradley's field data.

\section{B. Mean surface shear stresses}

Bradley plotted the mean surface shear stress measured downstream of the jump in roughness divided by the surface stress directly upstream of the jump. This ratio eventually relaxes downstream of the jump to the equilibrium value corresponding to the downstream surface. The ratio mainly depends on the two surface roughness values. The LES has to correctly predict this equilibrium ratio as well as the relaxation rate or the distance downstream of the jump where the ratio reaches its equilibrium value. Since LES codes with wall modeling "measure" the surface stress from the law-ofthe-wall using the velocity at the first grid point from the wall, they cannot accurately predict the departure from equilibrium of the stress ratio immediately after the roughness jump. This is due to the fact that the first grid points away from the wall do not lie in the internal equilibrium layer (IEL) of the downstream patch and hence are affected by the upstream patch. Since the first grid point is $5 \mathrm{~cm}(d z / 2)$ above the wall, one expects that the values of the stress ratio will be affected by this source of error up to $5 \mathrm{~m}$ downstream of the jump in roughness (about $5 \mathrm{~cm} \times 100$, see Brutsaer ${ }^{55}$ for scaling approximations for the IEL). Figures 10 and 11 depict the stress ratios measured by Bradley and simulated by the LES for low-to-high and high-to-low roughness transitions, respectively. The LASD model results agree well with the experimental results, especially past the downstream distance of $5 \mathrm{~m}$ after which the flow is in equilibrium with the underlying surface and the LES can accurately measure surface stress. The agreement between PASD model results and experimental data is slightly less satisfactory.

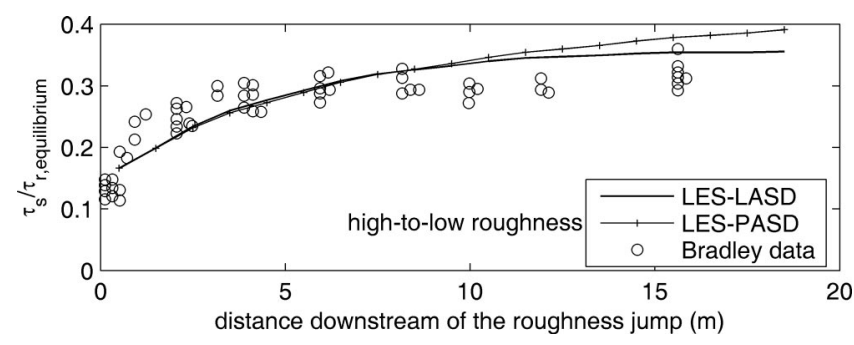

FIG. 11. Wall shear stress downstream of a high-to-low roughness jump normalized by the equilibrium stress of the upstream surface: comparison of LES results to Bradley's field data.

\section{Mean velocity profiles}

The streamwise velocity profiles produced by the LES are compared to the profiles measured by Bradley at various downstream distances. Following Bradley's approach, the velocities are normalized by the value at some height outside the internal boundary layer of the downstream patch $(220 \mathrm{~cm}$ for the low-to-high roughness transition and $112.5 \mathrm{~cm}$ for the high-to-low roughness transition). This is necessary since Bradley's data at different downstream distances are obtained at different times and hence cannot be normalized by $u^{*}\left(u^{*}\right.$ will not be constant at the various times the data are acquired). Figure 12 depicts the comparison results. For the low-to-high roughness transition, the two SGS models and the experimental data agree very well. For the high-to-low roughness transition, the two SGS models produce different results and the LASD data agrees better with the experimental data. This has been traced to a greater difference in SGS dissipation between the two models over the low-roughness surface (compared to the difference over the high-roughness surface) as will be shown in the following section.

\section{Coefficient variability over different patches and its effects}

In Fig. 13(a), the value of the Smagorinsky coefficient averaged in the cross-stream direction and in time, $\left\langle c_{s, \Delta}\right\rangle_{y, t}$, is plotted for the simulation with the LASD model reproducing Bradley's data for the high-to-low roughness transition. The coefficient is divided by its average over horizontal planes, $\left\langle c_{s, \Delta}\right\rangle_{x, y, t}$, to remove the effect of vertical variations and focus on horizontal variability. Up to $150 \%$ (minimum is $\sim 0.5$ of mean and maximum $\sim 1.3$ of mean) variations between the high-roughness and low-roughness patches can be observed near the ground, and the effect of surface heterogeneity extends well into the lowest $10 \%$ of the domain. This sensitivity of $c_{s, \Delta}$ to surface heterogeneity will not be captured by the planar-averaged model resulting in a difference in the SGS dissipation produced by the two models. This difference in estimating the SGS dissipation impacts the stress and velocity results produced by the models as depicted in Figs. 11 and 12. The dissipation of the LASD model divided by the dissipation of the PASD model is depicted in Fig. 13(b). It is clear that the difference is more significant and extends higher above the low roughness surface (compared to the difference above the high-roughness surface).

\section{E. Sensitivity to grid resolution}

Similar to the simulations over homogeneous surfaces, different grid resolutions were tested for the heterogeneous surfaces simulations reproducing Bradley's experiment. The results presented above pertain to the highest resolution used $(160 \times 64 \times 200$ nodes $)$. Two other resolutions were tested: a low resolution of $80 \times 32 \times 100$ nodes and a medium resolution of $120 \times 48 \times 150$ nodes. The results showed a consistent improvement in the reproduction of Bradley's experimental data as the grid resolution was increased; this applies for both the PASD and LASD subgrid scale models. In this section, we only present the results obtained with the LASD 

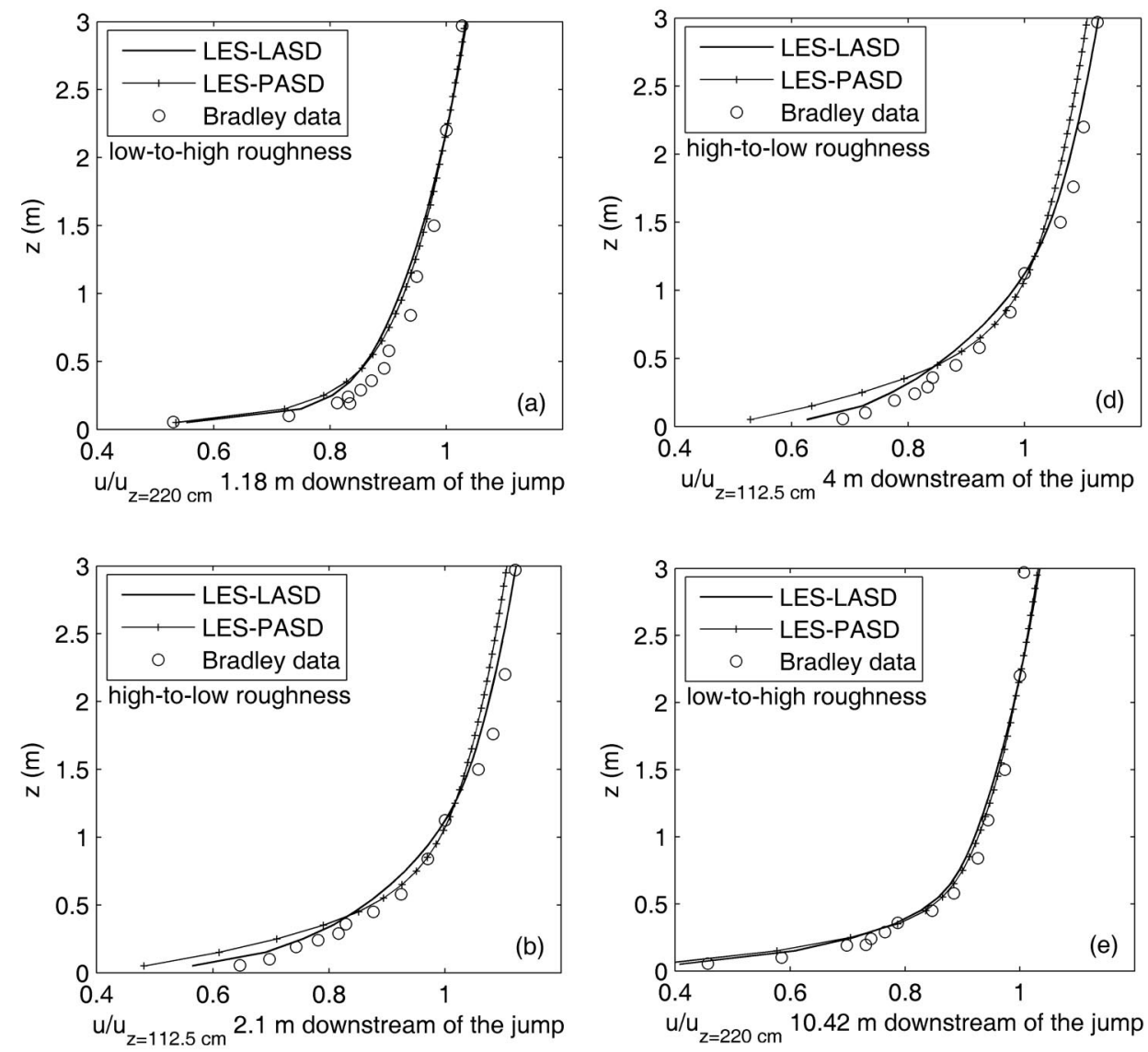

FIG. 12. Evolution of velocity profiles after an abrupt change in surface roughness: comparison of LES results to Bradley's field data.
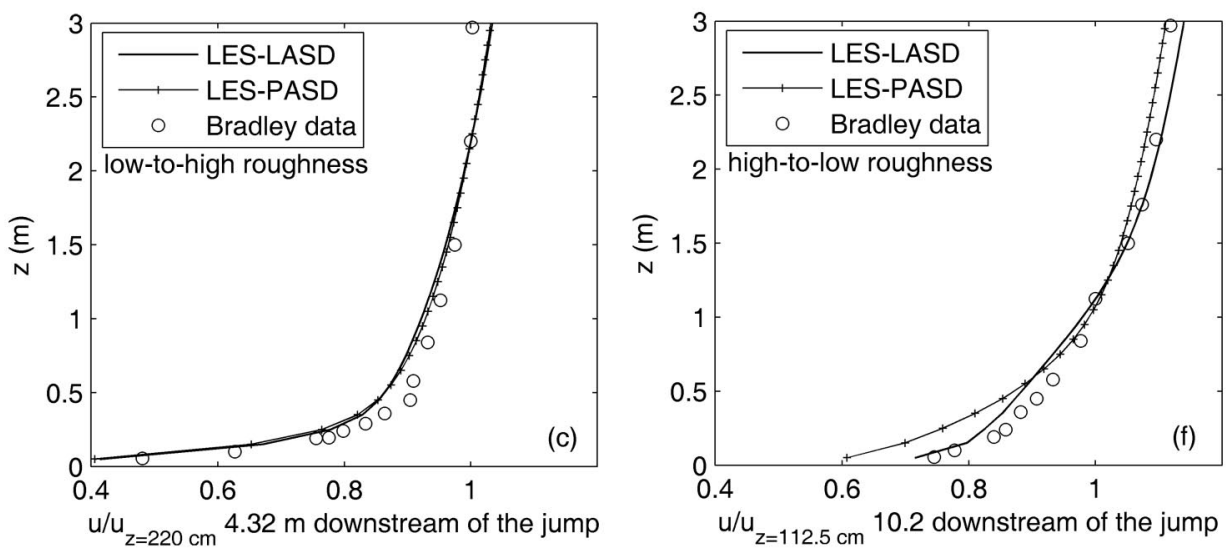

model. Figures 14 and 15 depict the wall shear stress downstream of a low-to-high and high-to-low roughness jumps, respectively. One observes that as the resolution decreases and the first grid point moves further away from the wall, the stress profile becomes flatter.

Figure 16 shows the velocity profile adjustment at about four meters downstream of the roughness jump. For the lowto-high transition, all resolutions are able to capture the experimental velocity profile rather well; this is in agreement with the results of the sensitivity to the SGS model. For this type of transition, the LES seems to be able to capture the velocity profile regardless of the numerical details and the SGS model. On the other hand, for the high-to-low roughness transition, the results from the low resolution simulation do not match the experimental data well. The improvement for the medium resolution is significant and little further improvement is obtained by passing to high resolution; this suggests that the results are converging to the experimental data as the resolution is increased. This type of roughness jump (high-to-low) is much more sensitive to the numerical details and the SGS model used.

\section{CONCLUSIONS}

A scale-dependent dynamic subgrid scale model has been formulated based on the Lagrangian time-averaging approach (LASD). The model extrapolates the Smagorinsky coefficient measured dynamically at two test-filter scales towards the grid-scale where the coefficient is unknown and needed for LES. Results show that the model performs well 

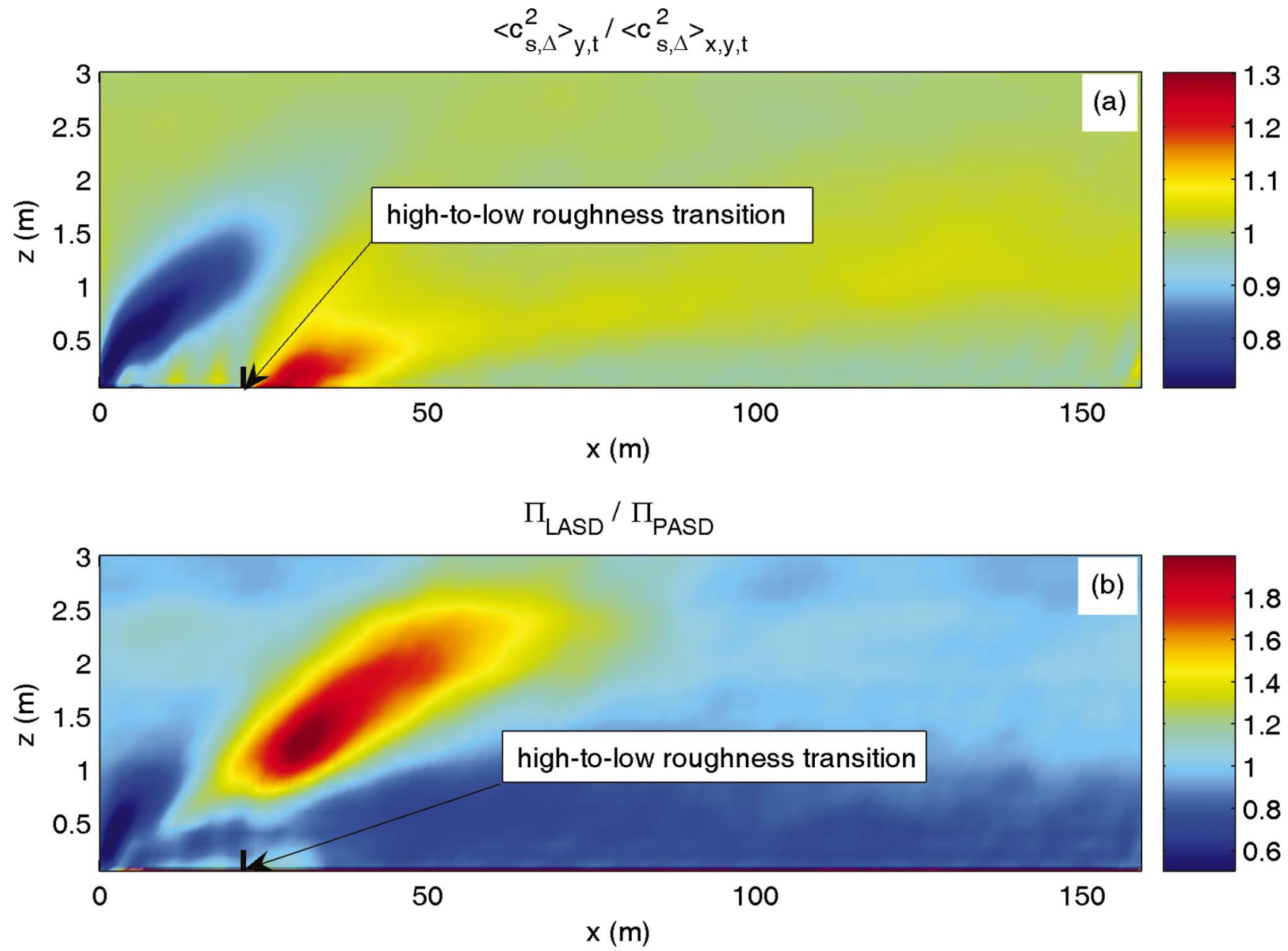

FIG. 13. (Color). Sensitivity of SGS model to surface roughness for a high-to-low roughness transition: (a) plot of the dynamic coefficient for the LASD model, (b) SGS dissipation predicted by the LASD model $\left(\Pi_{\text {LASD }}\right)$ divided by the SGS dissipation predicted by the PASD model ( $\left.\Pi_{\text {PASD }}\right)$.

in reproducing the log-law region in high-Reynolds number boundary layers that do not resolve the viscous sublayer, where scale-dependence is important. The model performs better than other SGS models tested here, including the Smagorinsky-Lilly model with a prescribed wall damping function, and the scale-invariant dynamic model. Streamwise velocity spectra indicate that the LASD model yields more accurate mean SGS dissipation properties. The model produces only a moderate increase in computational cost on the order of $20 \%$ (compared to the Smagorinsky model with



FIG. 14. Wall shear stress downstream of a low-to-high roughness jump, normalized by the equilibrium stress of the upstream surface: sensitivity to grid resolution for the LASD SGS model. HR is for high resolution (160 $\times 64 \times 200)$, MR is for medium resolution $(120 \times 48 \times 150)$, and $\operatorname{LR}$ is for low resolution $(80 \times 32 \times 100)$. imposed coefficient), since the dynamic coefficient need not be updated at every time step of the LES (here we updated it at every fifth time step, but this choice depends on CourantFriedricks-Lévy stability constraints of particular simulation parameters).

To test the model in an inhomogeneous flow under controlled conditions, it has been applied to LES to compare with the experimental field results of Bradley ${ }^{50}$ for highReynolds number boundary layer flow over a roughness discontinuity. The experimental results consist of stress and ve-

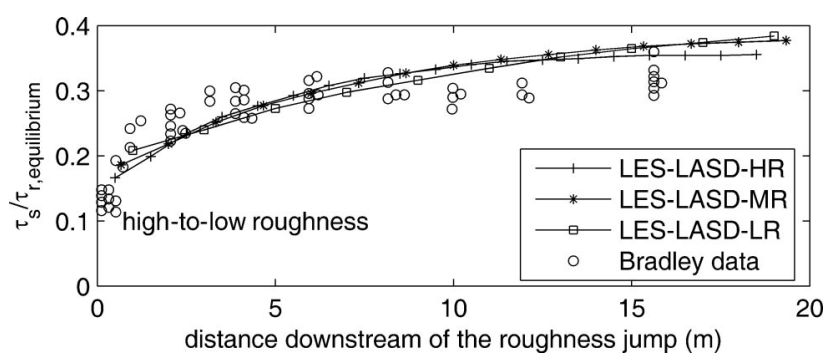

FIG. 15. Wall shear stress downstream of a high-to-low roughness jump normalized by the equilibrium stress of the upstream surface: sensitivity to grid resolution for the LASD SGS model. HR is for high resolution (160 $\times 64 \times 200)$, MR is for medium resolution $(120 \times 48 \times 150)$, and LR is for low resolution $(80 \times 32 \times 100)$. 

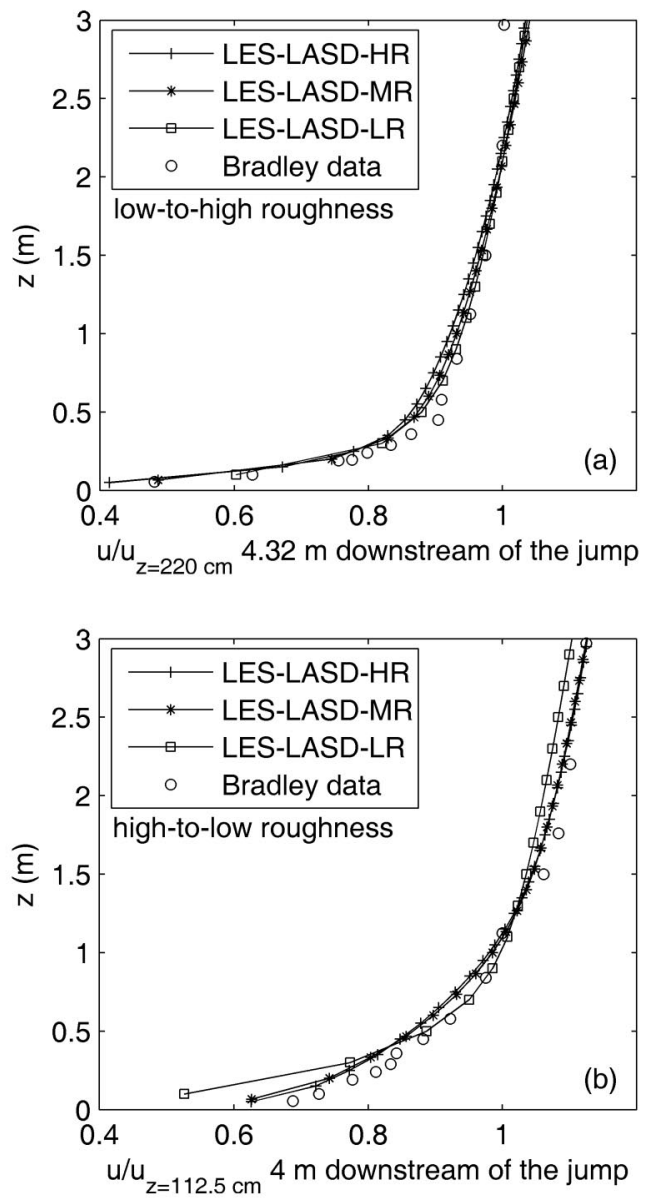

FIG. 16. Evolution of velocity profiles after an abrupt change in surface roughness: sensitivity to grid resolution for the LASD SGS model. HR is for high resolution $(160 \times 64 \times 200)$, MR is for medium resolution $(120 \times 48$ $\times 150)$, and LR is for low resolution $(80 \times 32 \times 100)$.

locity measurements downstream of the roughness jump. The LES was successful in predicting wall stress adjustment as a function of downstream distance. Similarly, the velocity profiles from LES data coincided well with the experimental data at several downstream distances included in the analysis. Results obtained with the Lagrangian model matched the experimental data better than results obtained with the equivalent planar-averaged model. This difference was traced back to the sensitivity of the Smagorinsky coefficient to the roughness height of the underlying surface. This sensitivity affects the SGS dissipation rate and cannot be captured by the planar-averaged formulation. This indicates that a local formulation, such as the Lagrangian one, is better suited for simulations of flows in complex geometries.

\section{ACKNOWLEDGMENTS}

This study was funded by NSF under Grant No. EAR9909679 and by Agreement No. R828771-0-01 from the U.S. Environmental Protection Agency's Science to Achieve Results (STAR) program. The authors are thankful to the Scientific Computing Division of the National Center for Atmospheric Research (NCAR) for the use of their computer clusters.

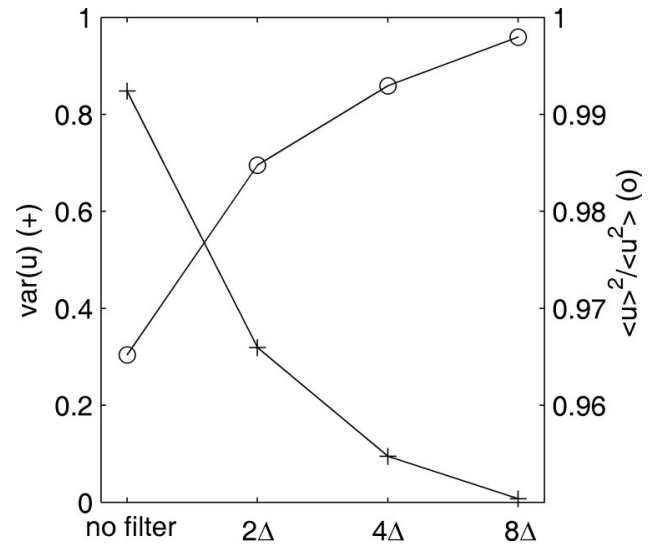

FIG. 17. Variance of $\overline{\tilde{u}}_{1}$ and ratio of $\left\langle\overline{\tilde{u}}_{1}\right\rangle^{2} /\left\langle\overline{\tilde{u}}_{1}^{2}\right\rangle$ as a function of filter size.

\section{APPENDIX: A LOCAL LAW-OF-THE-WALL FORMULATION}

In this appendix, an improved local law-of-the-wall is introduced. The common approach used in LES of highReynolds number boundary layer flow where the viscous sublayer is not resolved ${ }^{2,8,56,57}$ is to impose the law-of-thewall in a strictly local sense:

$$
\tau_{w}=-\left[\frac{\kappa}{\ln \left(z / z_{o}\right)}\right]^{2}\left(\widetilde{u}_{1}^{2}+\widetilde{u}_{2}^{2}\right) .
$$

Here $\kappa$ is the von-Karman constant $(\approx 0.4)$ and $z=d z / 2 . \tau_{w}$ is the kinematic stress $\tau / \rho$ (the squared friction velocity) at the wall. The use of this relation imposes an average stress obtained from LES:

$$
\left\langle\tau_{w}^{\mathrm{LES}}\right\rangle=-\left[\frac{\kappa}{\ln \left(z / z_{o}\right)}\right]^{2}\left(\left\langle\widetilde{u}_{1}^{2}\right\rangle+\left\langle\widetilde{u}_{2}^{2}\right\rangle\right)
$$

However, the log law was developed and validated to be used in an average sense, i.e.,

$$
\left\langle\tau_{w}^{\log }\right\rangle=-\left[\frac{\kappa}{\ln \left(z / z_{o}\right)}\right]^{2}\left\langle\widetilde{u}_{1}\right\rangle^{2},
$$

where the mean cross-stream component $\left\langle\widetilde{u}_{2}\right\rangle$ is zero. Since the velocity at $z=d z / 2$ fluctuates, $\left.\left\langle\widetilde{u}_{1}^{2}\right\rangle\right\rangle\left\langle\widetilde{u}_{1}\right\rangle^{2}$ (Schwartz inequality) and $\left.\left\langle\tau_{w}^{\mathrm{LES}}\right\rangle\right\rangle\left\langle\tau_{w}^{\mathrm{log}}\right\rangle$. Therefore, imposing the wall stress in a local formulation leads to increased average stresses for a given near-wall velocity. In LES with prescribed pressure gradient and mean stress, this would yield a slower flow near the surface.

A potential solution is to divide the stress into a mean contribution and a local contribution similar to the approach used to impose a velocity gradient at the surface. The local contribution should average to zero, yielding a proper estimate of the average stress. This formulation could be used in homogeneous terrain to impose a local stress. However, when complex or heterogeneous areas are to be simulated, defining the mean and the variation parts is not always feasible.

Fortunately, it turns out that filtering the velocity (at $z$ $=d z / 2$ and only to prescribe the wall stress) at a scale $2 \Delta$ already significantly reduces the small-scale fluctuations so 


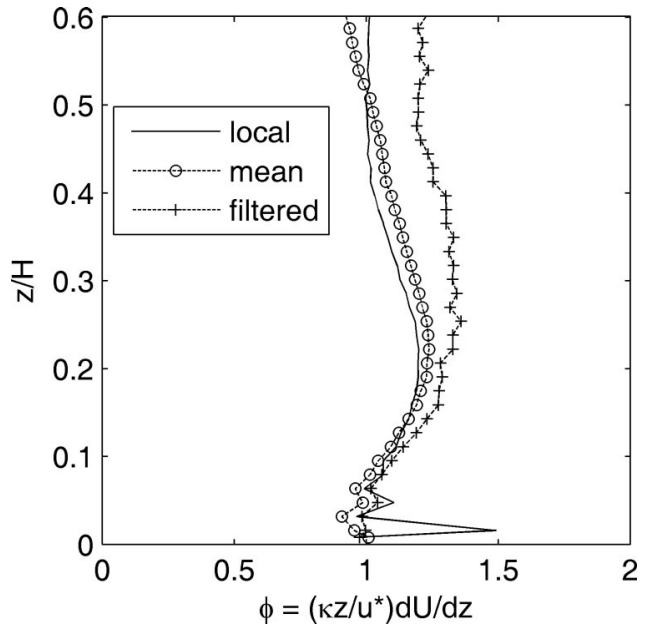

FIG. 18. Nondimensional velocity gradient for different wall model formulations.

that the velocity variance becomes quite small. A formulation that does not require averaging can be derived using this filtered local velocity to impose a stress as in Eqs. (34) and (35) of the main text.

The filtering preserves the large scale variations (larger than $2 \Delta$ ) which are important. At the same time, use of the filtered velocities results in an average stress that is very close to the stress predicted by the average similarity formulation for homogeneous surfaces. Figure 17 depicts the variance of the streamwise velocity $\tilde{u}$ and the ratio $\left\langle\tilde{\tilde{u}}_{1}\right\rangle^{2} /\left\langle\overline{\tilde{u}}_{1}^{2}\right\rangle$ for the unfiltered data (data at a scale $\Delta$ ) and for increasing filter size (obtained from our LASD simulations). A filtering at a scale of $2 \Delta$ is sufficient to increase $\overline{\tilde{u}}^{2} / \overline{\widetilde{u}}_{1}^{2}$ to about 0.985 . Note that there is no guarantee that this will yield a correct stress for heterogeneous surfaces. While a universal formulation to compute the average local or average stress over heterogeneous surfaces is yet to be found, this formulation can be implemented for heterogeneous surfaces and will certainly give better results that the fully local formulation.

Figure 18 presents the nondimensional velocity gradients for the different formulations, from simulations over homogeneous surfaces. The local formulation gives a very high gradient near the wall (and strong oscillations), this is related to the high stress and low velocity, predicted by this formulation, at the wall. On the other hand, the filtered formulation gives better results, as does the mean formulation which is known to be well suited for homogeneous cases.

${ }^{1}$ J. W. Deardorff, "Three-dimensional numerical study of the height and mean structure of a heated planetary boundary layer," Boundary-Layer Meteorol. 7, 81 (1974).

${ }^{2}$ C.-H. Moeng, "A large-eddy simulation for the study of planetary boundary layer turbulence," J. Atmos. Sci. 41, 2052 (1984).

${ }^{3}$ C.-H. Moeng and J. C. Wyngaard, "Statistics of conservative scalars in the convective boundary layer," J. Atmos. Sci. 41, 3161 (1984)

${ }^{4}$ R. H. Shaw and U. Schumann, "Large-eddy simulation of turbulent flow above and within a forest," Boundary-Layer Meteorol. 61, 47 (1992).

${ }^{5}$ P. J. Mason, "Large-eddy simulation: A critical review of the technique," Q. J. R. Meteorol. Soc. 120, 1 (1994).

${ }^{6} \mathrm{~B}$. Kosović, "Subgrid-scale modeling for the large-eddy simulation of high-Reynolds-number boundary layers," J. Fluid Mech. 336, 151 (1997).
${ }^{7}$ J. D. Albertson and M. B. Parlange, "Natural integration of scalar fluxes from complex terrain," Adv. Water Resour. 23, 239 (1999).

${ }^{8}$ J. D. Albertson and M. B. Parlange, "Surface length-scales and shear stress: implications for land-atmosphere interaction over complex terrain," Water Resour. Res. 35, 2121 (1999).

${ }^{9} \mathrm{M}$. Lesieur and O. Métais, "New trends in large eddy simulations of turbulence," Annu. Rev. Fluid Mech. 28, 45 (1996).

${ }^{10}$ U. Piomelli, "Large-eddy simulation: achievements and challenges," Prog. Aerosp. Sci. 35, 335 (1999).

${ }^{11}$ P. Sagaut, Large Eddy Simulation for Incompressible Flows, 2nd ed. (Springer, Berlin, 2003).

${ }^{12}$ J. S. Smagorinsky, "General circulation experiments with the primitive equations: I. The basic experiment," Mon. Weather Rev. 91, 99 (1963).

${ }^{13}$ J. Bardina, J. H. Ferziger, and W. C. Reynolds, "Improved subgrid-scale models for large-eddy simulation," AIAA Pap. 80, 1357 (1980).

${ }^{14}$ B. Vreman, B. Geurts, and H. Kuerten, "On the formulation of the dynamic mixed subgrid-scale model," Phys. Fluids 6, 4057 (1994).

${ }^{15}$ F. Sarghini, U. Piomelli, and E. Balaras, "Scale-similar models for largeeddy simulations," Phys. Fluids 11, 1596 (1999).

${ }^{16} \mathrm{R}$. Anderson and C. Meneveau, "Effects of the similarity model in finitedifference LES of isotropic turbulence using a Lagrangian dynamic mixed model," Flow, Turbul. Combust. 62, 201 (1999).

${ }^{17}$ C. Higgins, M. Parlange, and C. Meneveau, "Alignment trends of velocity gradients and subgrid-scale fluxes in the turbulent atmospheric boundary layer," Boundary-Layer Meteorol. 109, 59 (2003).

${ }^{18}$ H. S. Kang, S. Chester, and C. Meneveau, "Decaying turbulence in an active-grid-generated flow and comparisons with large-eddy simulation," J. Fluid Mech. 480, 129 (2003).

${ }^{19}$ M. Germano, U. Piomelli, P. Moin, and W. Cabot, "A dynamic subgridscale eddy viscosity model," Phys. Fluids A 3, 1760 (1991).

${ }^{20}$ M. Germano, "Turbulence: the filtering approach," J. Fluid Mech. 238, 325 (1992)

${ }^{21}$ C. Meneveau and J. Katz, "Scale-invariance and turbulence models for large-eddy simulation,” Annu. Rev. Fluid Mech. 32, 1 (2000).

${ }^{22}$ F. Porté-Agel, C. Meneveau, and M. B. Parlange, "A scale-dependent dynamic model for large-eddy simulation: application to a neutral atmospheric boundary layer," J. Fluid Mech. 415, 261 (2000).

${ }^{23}$ J. Kleissl, C. Meneveau, and M. B. Parlange, "On the magnitude and variability of subgrid-scale eddy-diffusion coefficients in the atmospheric surface layer," J. Atmos. Sci. 60, 2372 (2003).

${ }^{24}$ C. Meneveau, T. Lund, and W. Cabot, "A Lagrangian dynamic subgridscale model of turbulence," J. Fluid Mech. 319, 353 (1996).

${ }^{25}$ S. Ghosal, T. S. Lund, P. Moin, and K. Akselvoll, "A dynamic localization model for large eddy simulation of turbulent flows," J. Fluid Mech. 86, 229 (1995).

${ }^{26}$ S. B. Pope, Turbulent Flows (Cambridge University Press, Cambridge, UK, 2000).

${ }^{27}$ D. C. Haworth and K. Jansen, "Large-eddy simulation on unstructured deforming meshes: towards reciprocating IC engines," Comput. Fluids 29, 493 (2000).

${ }^{28}$ V. Armenio and U. Piomelli, "A Lagrangian mixed subgrid-scale model in generalized coordinates," Flow, Turbul. Combust. 65, 51 (2000).

${ }^{29}$ L. Blin, A. Hadjadj, and L. Vervisch, "Large eddy simulation of turbulent flows in reversing systems," J. Turbul. 4, 1 (2003).

${ }^{30}$ M. Tsubokura, T. Kobayashi, N. Taniguchi, and W. P. Jones, "A numerical study on the eddy structures of impinging jets excited at the inlet," Int. J. Heat Fluid Flow 24, 500 (2003).

${ }^{31} \mathrm{D}$. K. Lilly, "The representation of small scale turbulence in numerical simulation experiments," Proceedings of the IBM Scientific Computing Symposium on Environmental Sciences (IBM, Yorktown Heights, NY, 1967), p. 195.

${ }^{32}$ P. J. Mason and D. J. Thomson, "Stochastic backscatter in large-eddy simulations of boundary layers," J. Fluid Mech. 242, 51 (1992).

${ }^{33}$ D. K. Lilly, "A proposed modification of the Germano subgrid-scale closure method," Phys. Fluids A 4, 633 (1992).

${ }^{34} \mathrm{~S}$. B. Pope, "Ten questions concerning the large-eddy simulation of turbulent flows," New J. Phys. 6, 35 (2004).

${ }^{35} \mathrm{C}$. Meneveau and T. Lund, "The dynamic Smagorinsky model and scaledependent coefficients in the viscous range of turbulence," Phys. Fluids $\mathbf{9}$, 3932 (1997).

${ }^{36}$ E. Bou-Zeid, C. Meneveau, and M. B. Parlange, "Large-eddy simulation of neutral atmospheric boundary layer flow over heterogeneous surfaces: Blending height and effective surface roughness," Water Resour. Res. 40, W02505 (2004) 
${ }^{37}$ U. Piomelli and J. Liu, "Large-eddy simulation of rotating channel flows using a localized dynamic model," Phys. Fluids 7, 839 (1995).

${ }^{38} \mathrm{C}$. Meneveau and T. Lund, "On the Lagrangian nature of the turbulence energy cascade," Phys. Fluids 6, 2820 (1994).

${ }^{39}$ S. A. Orszag and Y. H. Pao, "Numerical computation of turbulent shear flows," Adv. Geophys. 18, 224 (1974).

${ }^{40}$ S. A. Orszag, "Transform method for calculation of vector coupled sums: application to the spectral form of the vorticity equation," J. Atmos. Sci. 27, 890 (1970).

${ }^{41}$ J. W. Deardorff, "A numerical study of three-dimensional turbulent channel flow at large Reynolds numbers," J. Fluid Mech. 41, 453 (1970).

${ }^{42}$ U. Piomelli and E. Balaras, "Wall-layer models for large-eddy simulation," Annu. Rev. Fluid Mech. 34, 349 (2002).

${ }^{43}$ A. Andren, A. R. Brown, J. Graf, P. J. Mason, C.-H. Moeng, F. T. M. Nieuwstadt, and U. Schumann, "Large-eddy simulation of a neutrally stratified boundary layer: a comparison of four computer codes," Q. J. R. Meteorol. Soc. 120, 1457 (1994).

${ }^{44}$ B. A. Kader and A. M. Yaglom, "Spectra and correlation functions of surface layer atmospheric turbulence in unstable thermal stratification," in Turbulence and Coherent Structures, edited by O. Métais and M. Lesieur (Kluwer Academic, Dordrecht, 1991) pp. 387-412.

${ }^{45}$ A. E. Perry, K. L. Lim, and S. M. Henbest, "An experimental study of the turbulence structure in smooth- and rough-wall boundary layers," J. Fluid Mech. 177, 437 (1987).

${ }^{46}$ G. G. Katul, C. R. Chu, M. B. Parlange, J. D. Albertson, and T. A. Ortenburger, "The low-wavenumber spectral characteristics of velocity and temperature in the atmospheric surface layer," J. Geophys. Res. 100, 14243 (1995).
${ }^{47}$ J. F. Morrison, W. Jiang, B. J. McKeon, and A. J. Smits, "Reynolds number dependence of streamwise velocity spectra in turbulent pipe flow," Phys. Rev. Lett. 88, 214501 (2002).

${ }^{48}$ T. B. Nickels and I. Marusic, "On the different contributions of coherent structures to the spectra of a turbulent round jet and a turbulent boundary layer," J. Fluid Mech. 448, 367 (2001).

${ }^{49}$ J. C. Del Alamo, J. Jimenez, P. Zandonade, and M. D. Moser, "Scaling of the energy spectra of turbulent channels," J. Fluid Mech. 500, 135 (2004).

${ }^{50}$ E. F. Bradley, "A micrometeorological study of velocity profiles and surface drag in the region modified by a change in surface roughness," Q. J. R. Meteorol. Soc. 94, 361 (1968).

${ }^{51}$ C. C. Shir, "A numerical computation of air flow over a sudden change of surface roughness," J. Atmos. Sci. 29, 304 (1972).

${ }^{52}$ K. S. Rao, J. C. Wyngaard, and O. R. Coté, "The structure of the twodimensional internal boundary layer over a sudden change of surface roughness," J. Atmos. Sci. 31, 738 (1974).

${ }^{53}$ W. H. Schofield, "Turbulent shear flows over a step-change in surface roughness," J. Fluids Eng. 103, 344 (1981).

${ }^{54}$ H. P. Schmid and B. Bünzli, "The influence of surface texture on the effective roughness length," Q. J. R. Meteorol. Soc. 121, 1 (1995).

${ }^{55} \mathrm{~W}$. Brutsaert, "Land-surface water vapor and sensible heat flux: Spatial variability, homogeneity, and measurement scale," Water Resour. Res. 34, 2433 (1998)

${ }^{56}$ P. J. Mason and N. S. Callen, "On the magnitude of subgrid-scale eddy coefficient in large-eddy simulations of turbulent channel flow," J. Fluid Mech. 162, 439 (1992).

${ }^{57}$ H. Schmidt and U. Schumann, "Coherent structures of the convective boundary layer derived from large-eddy simulations," J. Fluid Mech. 200, 511 (1989). 